Molecules 2004, 9, 300-322

molecules

ISSN 1420-3049

http://www.mdpi.org

\title{
From Labdanes to Drimanes. Degradation of the Side Chain of Dihydrozamoranic Acid.
}

\author{
Jesús M. L. Rodilla ${ }^{1, *}$, D. Díez ${ }^{2}$, J. G. Urones ${ }^{2}$ and Pedro M. Rocha ${ }^{1}$ \\ ${ }^{1}$ Departamento de Química, Universidade da Beira Interior, 6201-001 Covilhã, Portugal. Fax: \\ (+351) 275 319888; Tel: (+351) 275319700. \\ 2 Departamento de Química Orgánica, Facultad de Ciencias Químicas, Universidad de \\ Salamanca, 37008 Salamanca, Spain. Fax: (+34) 923 294574; Tel: (+34) 923 294474; E-mail: \\ ddm@usal.es
}

* Author to whom correspondence should be addressed; E-mail: rodilla@ubista.ubi.pt

Received: 23 February 2004; in revised form: 4 March 2004 / Accepted: 5 March 2004 / Published: 30 April 2004

\begin{abstract}
A new route for the degradation of the saturated side chain of dihydrozamoranic acid has been devised, giving an advanced intermediate, compound 14, useful for the synthesis of insect antifeedants such as warburganal and polygodial.
\end{abstract}

Keywords: Dihidrozamoranic acid, drimane derivatives, antifeedant.

\section{Introduction}

One of the main problems of our civilization is the shortage of food, especially in Third World countries. The intensity of the problem will increase as the global caloric demand is likely to double over the next ten years. This may be addressed in two ways; by better distribution of existing resources and by increasing total food supplies. With a rising productive area the number of pests has also increased considerably and thus the use of herbicides and insecticides [1]. Several insect species have developed resistance against agrochemicals causing not only a significant increase in the amount of chemicals used but also in the environmental pollutant level. The strategy used by plants to protect themselves from insect attack is the biosynthesis of secondary metabolites with antifeedant activity. 
These natural products are highly specific to some insect species and are completely inactive against other species useful to human beings [2]. Moreover, these compounds are biodegradable and there is no danger of accumulation or environmental pollution [3]. Among the natural antifeedants, azadirachtin isolated from Azadirachta indica [4], some clerodane diterpenoids such as jodrellin A and B isolated from Schutellaria woronowii Juss [5] and some drimanes such as warburganal (2b) isolated from Warburgia ugandensis [6] and polygodial (2a) isolated from Polygonum hydropiper [7] should be highlighted because their specific and high antifeedant activity against Spodoptera species [8], which causes more than 30\% crop losses in India [9]. In our lab several bioactive drimanes (Figure 1) have been synthetised starting from zamoranic acid [10].

\section{Figure 1}
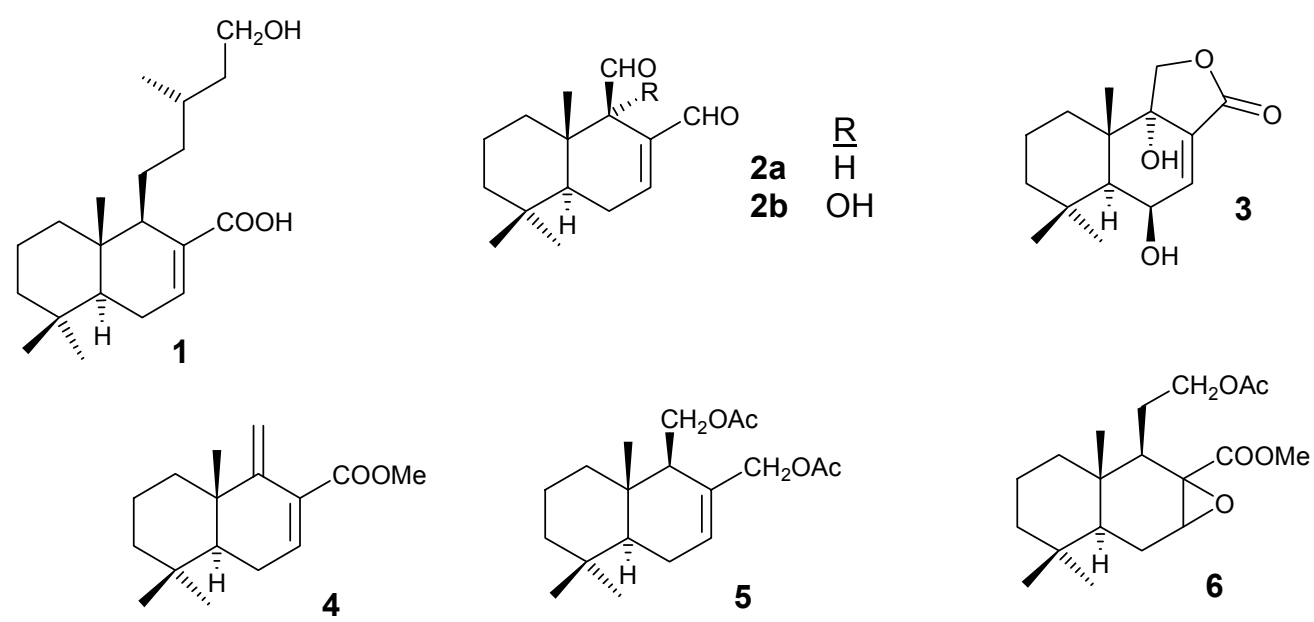

\section{Results and Discussion}

Dihydrozamoranic [11] acid (1), is a diterpene isolated from Halimium verticillatum and Halimium viscosum, being a main component in the first case. This compound's only difference with zamoranic acid is the saturation of the side chain. The transformation of this compound into an intermediate readily transformed in turn into natural compounds with biological activity would be of interest. Some of these intermediates are diene $\mathbf{4}$, the diacetylderivative 5 [12] and epoxide $\mathbf{6}$. The last compound has been transformed by us into poligodial (2a), warburganal (2b) and pereniporin A (3).

Starting from dihydrozamoranic acid methyl ester (1a), the retrosynthetic analysis as indicated below (Scheme 1), could be accomplished by two routes. In both cases the main degradation reaction is an elimination of the terminal carbon of the side chain, either by transformation into a double bond or by oxidative decarboxylation of an acid. 


\section{Scheme 1}<smiles>CC(=O)C1=CC[C@H]2[C@@H](C)CCC[C@]2(C)[C@H]1CC[C@H](C)CC(=O)O</smiles>

$\stackrel{\text { Route B }}{\longleftarrow}$<smiles>[R20]/C=C\C=C/[R6]</smiles>

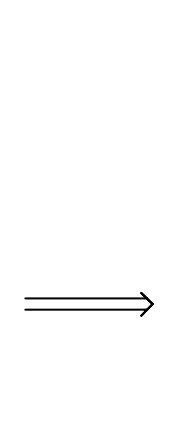<smiles>[R]COC(=O)COC(C)=O</smiles><smiles>[R]C12OC1C[C@H]1C(C)(C)CCC[C@]1(C)[C@@H]2CCC(C)=O</smiles>

Route A

The first route followed for the degradation of the side chain is shown in Scheme 2. Treatment of 1 a with $o$-nitrophenylselenocyanate $\left(\mathrm{nBu}_{3} \mathrm{P} / \mathrm{THF}\right)$ [13] gave an $87 \%$ yield of the nitrophenylselenyl derivative 7, which with $\mathrm{H}_{2} \mathrm{O}_{2}$ [14], produced a minor derivative 8 (4\%) and compound 9 in $92 \%$ yield. Treatment of 9 under Wacker conditions [15] with $\mathrm{CuCl} / \mathrm{PdCl}_{2} / \mathrm{O}_{2}$, lead to ketone $\mathbf{1 0}$ in $80 \%$ yield. Baeyer-Villiger oxidation [16] of $\mathbf{1 0}$ with m-CPBA gives 11, a 1:2 mixture of $\alpha$ and $\beta$ epoxides, in 95\% yield. This compound shows a degradation of the side chain by two carbons.

The hydrolysis of 11 with $\mathrm{K}_{2} \mathrm{CO}_{3} / \mathrm{MeOH}$ gives 12 (85\% yield), a mixture of epoxy alcohols that by oxidation with $\mathrm{CrO}_{3} / \mathrm{Py}$ [17] gave the epoxyketones 13 (82\%). As in previous studies, the degradation of the nor-derivatives to the drimane skeleton was done by Norrish II type reaction [18]. Photochemical treatment (hv; $\lambda=366 \mathrm{~nm}$ ) of 13 lead to olefins $14(9.5 \%)$ and $15(22.5 \%)$, while a $(39 \%)$ of 13 was recovered, so the global yield is quite good when the recovery of the starting material is taken into account. 


\section{Scheme 2.}

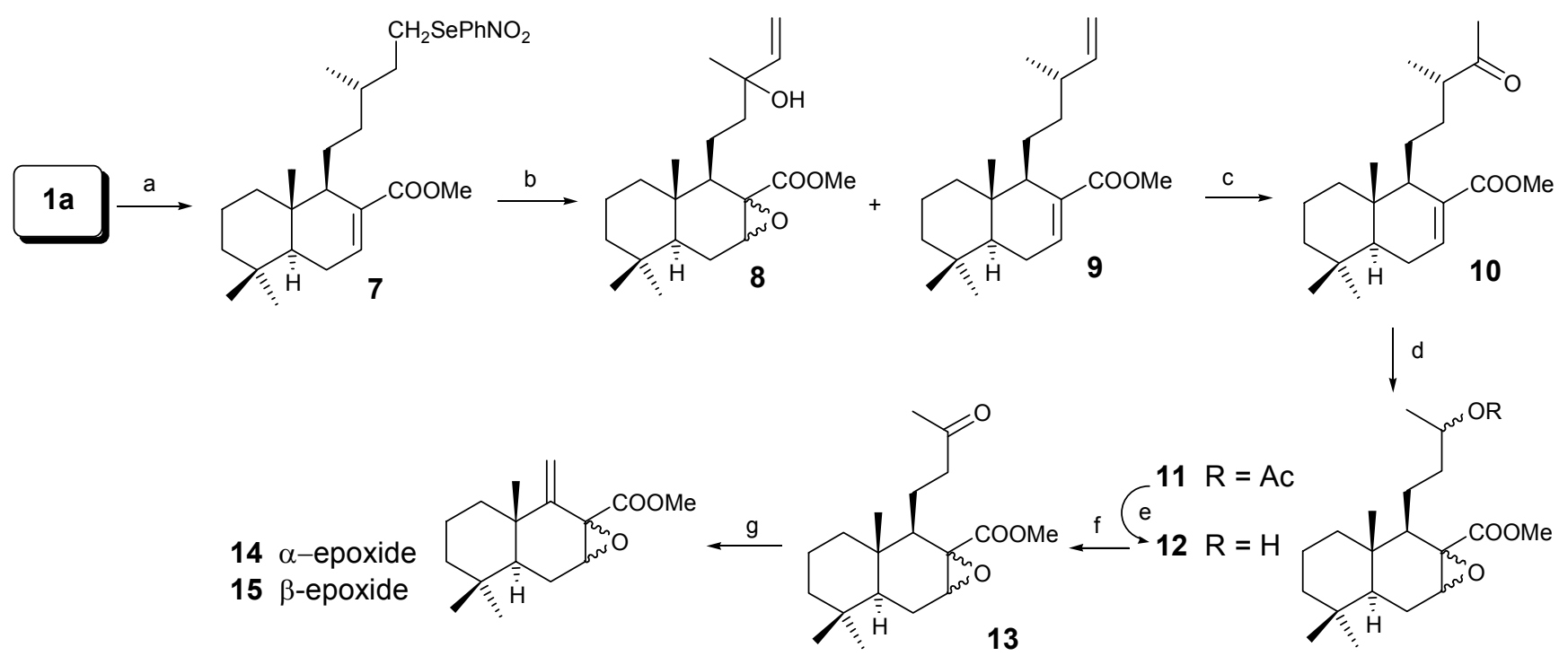

Reagents: (a) $o-\mathrm{NO}_{2} \mathrm{PhSeCN} / \mathrm{nBu}_{3} \mathrm{P} / \mathrm{THF}$; (b) $\mathrm{H}_{2} \mathrm{O}_{2} / \mathrm{TFH}$; (c) $\mathrm{CuCl} / \mathrm{PdCl}_{2} / \mathrm{O}_{2} / \mathrm{DMF}$;

(d) $m$-CPBA/ $\mathrm{CH}_{2} \mathrm{Cl}_{2}$; (e) $\mathrm{K}_{2} \mathrm{CO}_{3} / \mathrm{MeOH}$; (f) $\mathrm{CrO}_{3} / \mathrm{Py}$; (g) Norrish II

The synthesis of compound $\mathbf{1 1}$ could be achieved by epoxidation and then degradation, as shown in Scheme 3. Treatment of dihydrozamoranic acid methyl ester (1a) with $m$-CPBA led to a $67 \%$ yield of the mixture of epoxides 16 in a ratio 3:2 ( $\alpha: \beta)$, which by treatment with $o$-nitrophenylselenocyanate gives the mixture 17 in $75 \%$ yield. Oxidation of 17 with $\mathrm{H}_{2} \mathrm{O}_{2}$ lead to the $\Delta^{14}$ olefin $\mathbf{1 8}$, in $86 \%$ yield. Wacker oxidation with $\mathrm{CuCl} / \mathrm{PdCl}_{2}$ and $\mathrm{O}_{2}$ gave the corresponding ketone, 19 (80\%). Baeyer-Villiger oxidation with $m$-CPBA gave 11 (94\% yield).

\section{Scheme 3.}

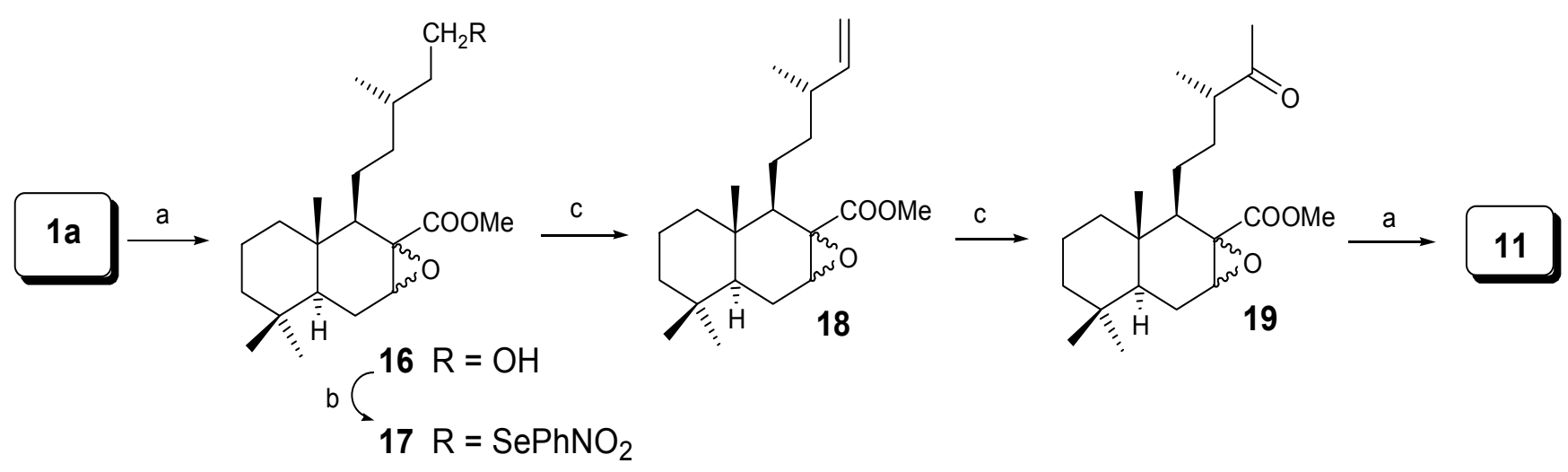

Reagents: (a) $m$-CPBA/ $\mathrm{CH}_{2} \mathrm{Cl}_{2}$; (b) $o-\mathrm{NO}_{2} \mathrm{PhSeCN} / \mathrm{nBu}_{3} \mathrm{P} / \mathrm{THF}$; (c) $\mathrm{H}_{2} \mathrm{O}_{2} / \mathrm{THF}$;

(d) $\mathrm{CuCl} / \mathrm{PdCl}_{2} / \mathrm{O}_{2} / \mathrm{DMF}$. 
Compound 13 can also be obtained in a different manner, starting from 20, a natural compound isolated from $H$. verticillatum and $H$. viscosum [19] (Scheme 4), which in a similar manner as described before was transformed into compound 24. Hydrolysis of $\mathbf{2 4}$ under basic conditions gave $\mathbf{2 5}$, the epoxide of Payne rearrangement [20] 26 and 27 that were separated by CC. Compound $\mathbf{2 5}$ was oxidized to give 13, previously transformed into drimanes 14/15 (Scheme 2).

\section{Scheme 4}

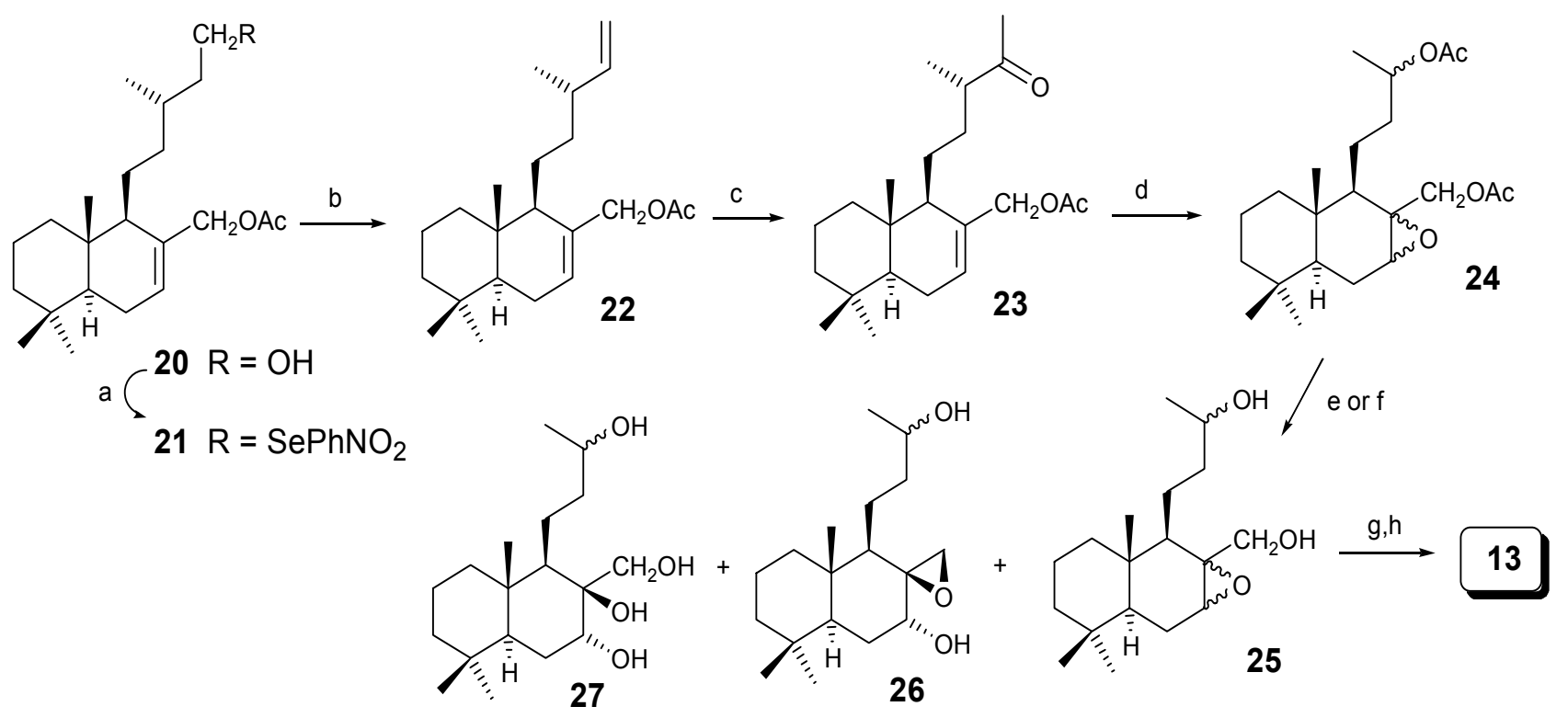

Reagents: (a) $o-\mathrm{NO}_{2} \mathrm{PhSeCN} / \mathrm{nBu}_{3} \mathrm{P} / \mathrm{THF}$; (b) $\mathrm{H}_{2} \mathrm{O}_{2} / \mathrm{THF}$; (c) $\mathrm{CuCl} / \mathrm{PdCl}_{2} / \mathrm{O}_{2} /$ DMF; (d) $m$-CPBA/ $\mathrm{CH}_{2} \mathrm{Cl}_{2}$; (e) $\mathrm{K}_{2} \mathrm{CO}_{3} / \mathrm{MeOH}$; (f) $\mathrm{NaOH} / \mathrm{MeOH}$; (g) $\mathrm{CrO}_{3} / \mathrm{AcOH}$; (h) $\mathrm{CH}_{2} \mathrm{~N}_{2} /$ ether.

Route B

A second approach is based on the degradation of the side chain by an oxidative decarboxylation process (Scheme 5). The synthesis of the acid group on C-15 and epoxide C-7 can be acheived in two different ways. Oxidation of $1 \mathrm{a}$ with $\mathrm{CrO}_{3} / \mathrm{AcOH}$ gives acid $\mathbf{2 8}$ in $60 \%$ yield, which by treatment with $m$-CPBA lead to the $3: 2$ mixture of $\alpha$ and $\beta$ epoxides 29 in $60 \%$ yield. Alternatively, treatment of 1a with $m$-CPBA gives the 3:2 mixture of $\alpha$ and $\beta$ epoxides 16, then oxidation with PDC/DMF [21] or $\mathrm{CrO}_{3} / \mathrm{AcOH}$ lead to the same $3: 2$ mixture of $\alpha$ and $\beta$ epoxides 29 , (in $74 \%$ and $56 \%$ yields) respectively. 


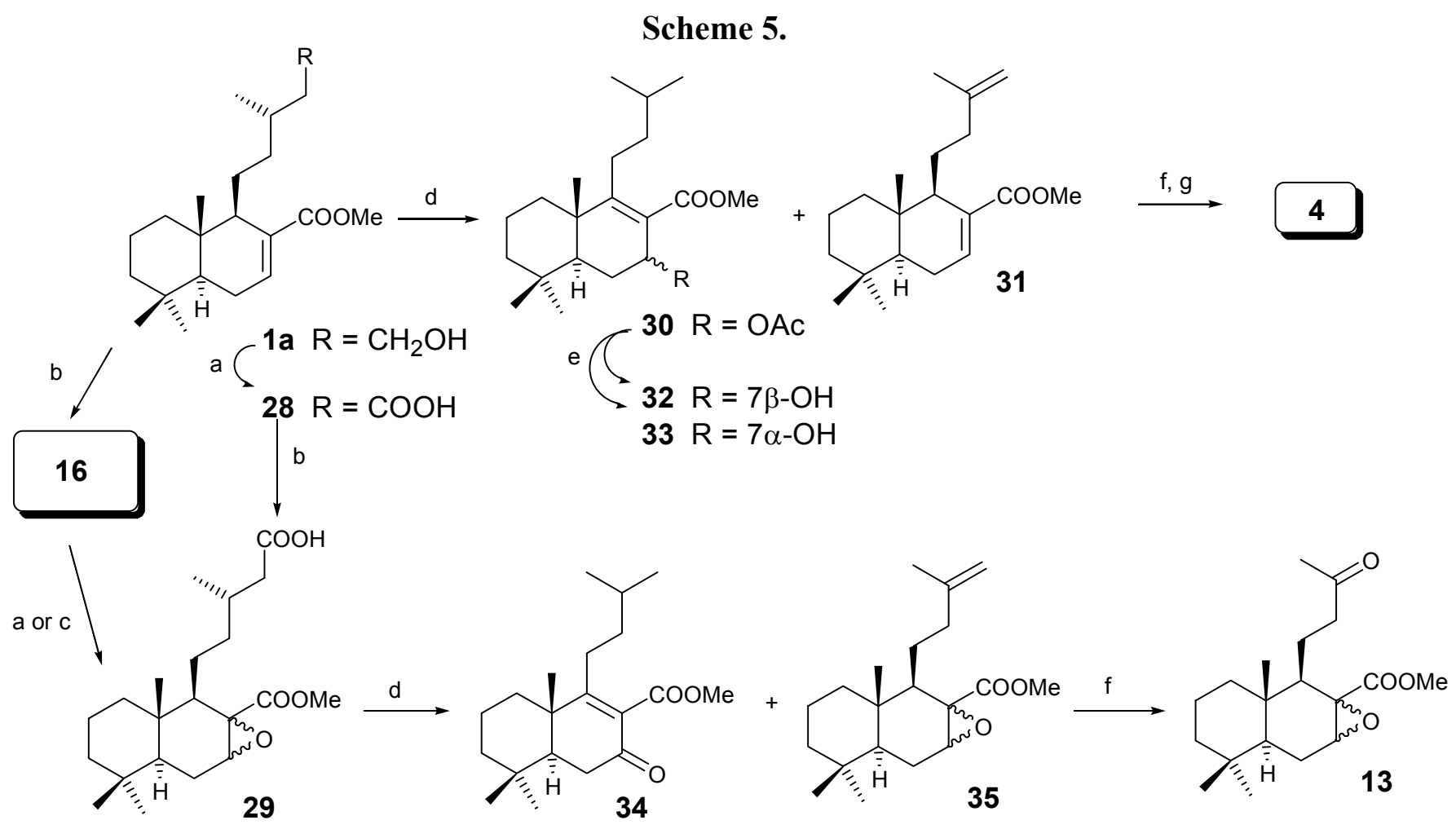

Reagents: (a) $\mathrm{CrO}_{3} / \mathrm{AcOH}$; (b) $m$-CPBA/ $\mathrm{CH}_{2} \mathrm{Cl}_{2}$; (c) $\mathrm{PDC} / \mathrm{DMF}$; (d) $(\mathrm{AcO})_{4} \mathrm{~Pb} /$ $(\mathrm{AcO})_{2} \mathrm{Cu} / \mathrm{Py} / \mathrm{C}_{6} \mathrm{H}_{6}$; (e) $\mathrm{K}_{2} \mathrm{CO}_{3} / \mathrm{MeOH}$; (f) $\mathrm{O}_{3} / \mathrm{CH}_{2} \mathrm{Cl}_{2}$; (g) Norrish II

Oxidative decarboxylation of $\mathbf{2 8}$ gave $\mathbf{3 0}$ and $\mathbf{3 1}$ in $40 \%$ and $8 \%$ yield, respectively. The major component was hydrolysed to give alcohols 32 and 33. It is very interesting to note that the decarboxylation takes place giving mainly an isobutyl group in the parent compound $\mathbf{3 0}$.

The minor compound $\mathbf{3 1}$ was transformed by ozonolysis and Norrish II type reaction into diene 4, already transformed into active drimanes [12]. As the yield was very poor it was decided to do the same reaction with compound 29, leading again, to a major component 34 (62\%) and a terminal olefin $35(12 \%)$ which was transformed into epoxides 13.

As we have seen with $m$-CPBA, a mixture of epoxides at C-7 was always obtained. In order to obtain only one epoxide, dihydrozamoranic acid methyl ester (1a) was treated with dimethyldioxirane [23], giving selectively only compound $29 \alpha$ in $45 \%$ yield and producing the oxidation of the primary alcohol into the acid at C-15; following the same sequence as in Scheme 6 only compound 14 was obtained. 


\section{Scheme 6}

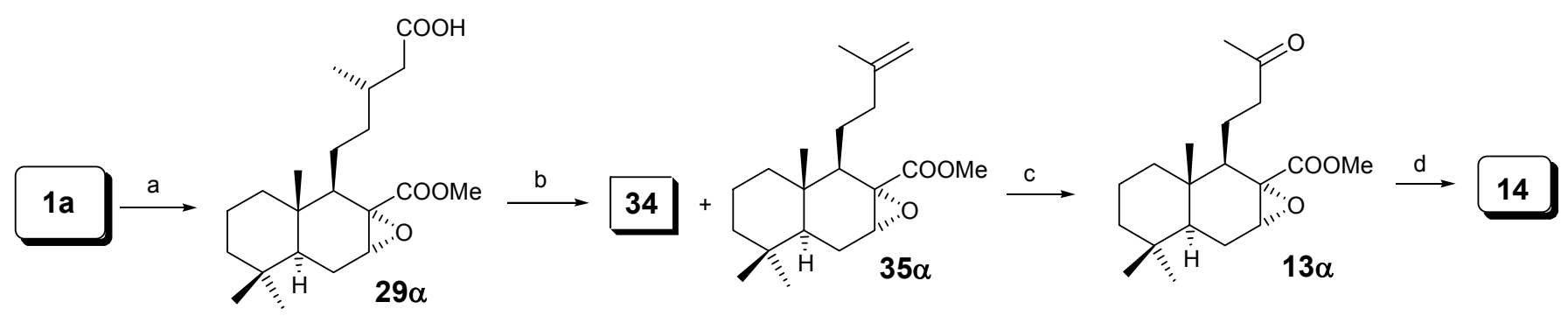

Reagents: (a) dimethyldioxirane / acetone; (b) $(\mathrm{AcO})_{4} \mathrm{~Pb} /(\mathrm{AcO})_{2} \mathrm{Cu} / \mathrm{Py} / \mathrm{C}_{6} \mathrm{H}_{6}$;

(c) $\mathrm{O}_{3} / \mathrm{CH}_{2} \mathrm{Cl}_{2} ;$ (d) Norrish II

\section{Conclusions}

We have developed a new procedure to obtain drimane $\mathbf{1 4}$ from dihydrozamoranic acid, making use of methodology developed in our laboratory [24] that could be useful for further transformations.

\section{Acknowledgments}

The authors are grateful to A. Lithgow, Servicio General de Resonancia Magnética Nuclear, Facultad de Ciencias Químicas, Universidad de Salamanca for the NMR spectra.

\section{Experimental}

\section{General}

Unless otherwise stated, all chemicals were purchased as the highest purity commercially available and were used without further purification. IR spectra (thin film) were recorded on a MATTSONGENESIS II FT-IR spectrophotometer. ${ }^{1} \mathrm{H}$ - and ${ }^{13} \mathrm{C}$-NMR spectra were recorded in deuterochloroform and referenced to the residual peak of $\mathrm{CHCl}_{3}$ at $\delta 7.26 \mathrm{ppm}$ and $\delta 77.0 \mathrm{ppm}$, for ${ }^{1} \mathrm{H}-$ and ${ }^{13} \mathrm{C}$-, respectively, on a Bruker WP-200 SY or a Bruker DRX $400 \mathrm{MHz}$ instrument. Chemical shifts are reported in $\delta \mathrm{ppm}$ and coupling constants $(\mathrm{J})$ are given in Hz. MS were performed at a VG-TS 250 spectrometer at $70 \mathrm{eV}$ ionising voltage. Mass spectra are represented at $m / z$ (\% rel. int.). HRMS were recorded on a VG Platform (Fisons) spectrometer using chemical ionisation (ammonia as gas). Optical rotations were determined at a digital ADP 220 polarimeter in $1 \mathrm{dm}$ cells. Diethyl ether, THF and benzene were distilled from sodium, and pyridine and dichloromethane were distilled from calcium hydride under an Ar atmosphere. The raw material 1 was isolated from a hexane extract of Halimium verticillatum as reported in reference [11]. 
Reaction of 1a: Synthesis of methyl 15-o-nitrophenylseleno-7-labden-17-oate (7).

To a solution of 1a $(750 \mathrm{mg}, 2.2 \mathrm{mmol})$ in dry THF $(3.3 \mathrm{~mL})$ was added $o$-nitrophenylselenocyanate $(635.8 \mathrm{mg}, 2.8 \mathrm{mmol})$ and the mixture was stirred under an inert atmosphere for $20 \mathrm{~min}$. at $48^{\circ} \mathrm{C} . \mathrm{nBu}_{3} \mathrm{P}(504.5 \mathrm{mg}, 2.5 \mathrm{mmol})$ was added to the reaction mixture, that was stirred for $48 \mathrm{~min}$, then the solvent was removed and the residue chromatographed (95:5 hexane/EtOAc) yielding 652.5 mg (87\%) of 7; ${ }^{1} \mathrm{H}-\mathrm{NMR} \delta: 8.30$ (d, $1 \mathrm{H}, \mathrm{J}=8.2, \mathrm{H}-3$ '), 7.47 and 7.27 (both $\mathrm{m}, 3 \mathrm{H}, \mathrm{H}-4$ ', H-5' and H6'), 6.66 (m, 1H, H-7), 3.70 (s, 3H, MeOOC), 2.95 (m, 2H, H-15), $2.15-1.30$ (m, 11H), $1.22-0.90$ (m, 6H), 0.98 (d, 3H, J=5.8 Hz, Me-16), 0.92 (s, 3H, Me-19), 0.89 (s, 3H, Me-18) and 0.84 (s, 3H, Me20); ${ }^{13} \mathrm{C}-\mathrm{NMR} \delta: 39.4$ (C-1), 18.4 (c-2), 41.9 (C-3), 32.6 (C-4), 49.3 (C-5), 23.8 (C-6), 137.0 (C-7), 135.2 (C-8), 50.9 (C-9), 36.8 (C-10), 25.6 (C-11), 37.8 (C-12), 34.3 (C-13), 34.7 (C-14), 23.8 (C-15), 19.3 (C-16), 169.6 (C-17), 33.0 (C-18), 21.8 (C-19), 14.3 (C-20), 51.2 (COOMe), 134.0 (C-1'), 146.3 (C-2'), 126.3 (C-3'), 128.9 (C-4'), 133.4 (C-5'), 125.1 (C-6'); IR cm ${ }^{-1}$ : 2925, 1714, 1644, 1590, 1564 , $1515,1332,1247,1068,730$.

Oxidation of 7 with $\mathrm{H}_{2} \mathrm{O}_{2}$ : Synthesis of methyl 13-hydroxy-7,14-labdadien-17-oate (8) and methyl 7,14-labdadien-17-oate (9).

To a solution of $7(600 \mathrm{mg}, 1.15 \mathrm{mmol})$ in THF $(6 \mathrm{~mL})$ was added $30 \% \mathrm{H}_{2} \mathrm{O}_{2}(0.30 \mathrm{~mL}, 130 \mathrm{mg}$, $2.0 \mathrm{mmol}$ ) and the mixture was stirred for $12 \mathrm{~h}$. The solvent was evaporated at reduced pressure and the crude product chromatographed eluting with a hexane/EtOAc gradient yielding $24 \mathrm{mg}(4 \%)$ of $\mathbf{8}$ (95:5 hexane/EtOAc) and $552 \mathrm{mg}(92 \%)$ of 9 (98:2 hexane/EtOAc).

Compound 8: ${ }^{1} \mathrm{H}-\mathrm{NMR} \delta: 6.69$ (m, 1H, H-7), 5.86 (dd, 1H, J=17.4, 10.7, H-14), 5.19 (d, 1H, $\mathrm{J}=17.4, \mathrm{H}-15 \mathrm{a}), 5.02$ (d, 1H, J=17.4, H-15b), 3.69 (s, 3H, COOMe), $2.25-1.35$ (m, 8H), $1.25-0.90$ (m, 6H), 1.22 (s, 3H, Me-16), 0.89 (s, 3H, Me-19), 0.85 (s, 3H, Me-18) and 0.80 (s, 3H, Me-20); ${ }^{13} \mathrm{C}-\mathrm{NMR}$ 8: 39.3 (C-1), 18.4 (C-2), 41.9 (C-3), 32.6 (C-4), 49.3 (C-5), 22.0 (C-6), 137.4 and 137.6 (C7, epimers), 135.5 and 135.7 (C-8, epimers), 50.7 and 50.8 (C-9, epimers), 36.9 (C-10), 23.9 (C-11), 42.6 and 42.8 (C-12, epimers), 73.1 (C-13), 144.9 and 145.4 (C-14, epimers), 111.3 and 111.6 (C-15, epimers), 169.5 (C-17), 33.0 (C-18), 21.9 (C-19), 14.2 (C-20), 51.3 (COOMe); IR cm ${ }^{-1}$ : 3446, 3086, 2924, 1717, 1646, 1245,1071, 917; MS m/z $\left(\mathrm{EI}^{+}\right): 334\left(\mathrm{M}^{+},<1 \%\right), 316$ (4), 284 (19), 249 (15), 248 (79), 233 (28), 203 (15), 192 (29) 175 (25), 124 (15), 109 (56), 105 (16), 91 (24), 81 (33), 79 (34), 77 (15), 71 (100), 69 (28), 67 (20), 59 (15), 55 (45); HRMS (CI) for $\mathrm{C}_{21} \mathrm{H}_{34} \mathrm{O}_{3}\left(\mathrm{MH}^{+}\right)$: Calc 334.2508; Found 334.2519 .

Compound 9: ${ }^{1} \mathrm{H}-\mathrm{NMR} \delta: 6.51$ (m, 1H, H-7), 5.56 (ddd, $\left.1 \mathrm{H}, \mathrm{J}=17.3,10.1,7.3, \mathrm{H}-14\right), 4.82$ (dd, $1 \mathrm{H}$, $\mathrm{J}=17.3 \mathrm{~Hz}, 2.1 \mathrm{~Hz}, \mathrm{H}-15 \mathrm{a}), 4.78$ (dd, 1H, J=10.1, $2.1 \mathrm{~Hz}, \mathrm{H}-15 \mathrm{~b}$ ), 3.59 (s, 3H, COOMe), $2.15-1.65$ (m, 4H), $1.60-1.25(\mathrm{~m}, 6 \mathrm{H}), 1.20-0.90(\mathrm{~m}, 5 \mathrm{H}), 0.87$ (d, 3H, J=6.7, Me-16), 0.81 (s, 3H, Me-19), 0.77 (s, 3H, Me-18) and 0.72 (s, 3H, Me-20); ${ }^{13} \mathrm{C}-\mathrm{NMR}$ 8: 39.4 (C-1), 18.3 (C-2), 41.9 (C-3), 32.5 (C- 
4), 49.2 (C-5), 23.6 (C-6), 136.2 (C-7), 135.4 (C-8), 50.8 (C-9), 36.6 (C-10), 25.7 (C-11), 37.8 (C-12), 38.5 (C-13), 144.4 (C-14), 112.2 (C-15), 20.1 (C-16), 169.3 (C-17), 33.0 (C-18), 21.8 (C-19), 14.2 (C20), 50.9 (COOMe); $[\alpha]_{\mathrm{D}}{ }^{20}-16.7$ (c 0.9 in $\mathrm{CHCl}_{3}$ ); IR cm${ }^{-1}: 3076,2951,1722,1643,1434,1246$, 1066, 909; MS m/z (EI $) 318$ (M+1 1.8\%), 248 (10), 195 (3), 194 (9), 165 (3), 162 (13), 137 (8), 135 (12), 124 (79), 109 (100), 95 (36), 81 (41), 69 (50), 67 (34), 55 (83).

Wacker oxidation of 9: Synthesis of methyl 14-oxo-7-labden-17-oate (10).

A solution of $\mathrm{PdCl}_{2}(128 \mathrm{mg}, 0.72 \mathrm{mmol})$ and $\mathrm{CuCl}(3.5 \mathrm{~g}, 3.6 \mathrm{mmol})$ in $\mathrm{DMF}(10 \mathrm{~mL})$ and $\mathrm{H}_{2} \mathrm{O}(1$ $\mathrm{mL})$ was activated for $30 \mathrm{~min}$ with $\mathrm{O}_{2}$. A solution of $9(1.1 \mathrm{~g}, 3.6 \mathrm{mmol})$ in DMF $(8 \mathrm{~mL})$ was added and stirred with $\mathrm{O}_{2}$ atmosphere at room temperature for $36 \mathrm{~h}$. Then to this mixture was added a cooled solution of $3 \mathrm{~N} \mathrm{HCl}$ and the mixture was extracted with $\mathrm{CH}_{2} \mathrm{Cl}_{2}(3 \times 30 \mathrm{~mL})$ and washed successively with $10 \% \mathrm{NaHCO}_{3}$ and water, dried, filtered, evaporated and chromatographed (9:1 hexane/EtOAc) to give $910 \mathrm{mg}(80 \%)$ of $10 ;{ }^{1} \mathrm{H}-\mathrm{NMR} \delta: 6.45$ (m, $\left.1 \mathrm{H}, \mathrm{H}-7\right), 3.49$ (s, 3H, COOMe), 2.24 (sex, $1 \mathrm{H}, \mathrm{J}=6.8$, H-13), $2.00-1.60$ (m, 4H), 1.91 (s, 3H, Me-15), $1.40-0.90$ (m, 10H), 0.85 (d, 3H, J=6.8, Me-16), 0.69 (s, 3H, Me-19), 0.65 (s, 3H, Me-18) and 0.60 (s, 3H, Me-20); ${ }^{13} \mathrm{C}-\mathrm{NMR} \delta: 39.0$ (C-1), 18.1 (C-2), 41.5 (C-3), 32.3 (C-4), 48.9 (C-5), 23.5 (C-6), 136.8 (C-7), 134.6 (C-8), 50.5 (C-9), 36.4 (C-10), 25.4 (C-11), 34.1 (C-12), 47.6 (C-13), 211.8 (C-14), 27.1 (C-15), 15.6 (C-16), 168.7 (C-17), 32.7 (C-18), 21.5 (C-19), 13.9 (C-20), 50.8 (COOMe); $[\alpha]_{\mathrm{D}}{ }^{20}-11.1$ (c 0.2 in $\mathrm{CHCl}_{3}$ ); IR cm ${ }^{-1}: 2927,1713,1645$, 1461, 1365, 1254, 1065; MS m/z (EI $) 334\left(\mathrm{M}^{+},<0.8 \%\right.$ ), 302 (2), 263 (5), 211 (8), 179 (37), 124 (32), 109 (100), 105 (20), 95 (16), 91 (32), 81 (26), 79 (32), 77 (17), 69 (26), 67 (17), 59 (12), 55 (30); HRMS (CI) for $\mathrm{C}_{21} \mathrm{H}_{34} \mathrm{O}_{3}\left(\mathrm{MH}^{+}\right)$: Calcd 334.2508; Found 334.2517.

Baeyer-Villiger reaction and epoxidation of $\mathbf{1 0}$ with m-CPBA: Synthesis of methyl 13-acetoxy-7,8epoxy-14,15-dinor-labdan-17-oate (11).

To a solution of $10(334.5 \mathrm{mg}, 1.0 \mathrm{mmol})$ in dry $\mathrm{CH}_{2} \mathrm{Cl}_{2}(5 \mathrm{~mL}), m$-CPBA $(345.0 \mathrm{mg}, 2.0 \mathrm{mmol})$ was added and the mixture stirred at room temperature. After $12 \mathrm{~h}$, the solvent was removed and ether was added. The organic phase was washed with $40 \% \mathrm{Na}_{2} \mathrm{~S}_{2} \mathrm{O}_{3}, 10 \% \mathrm{Na}_{2} \mathrm{CO}_{3}$ and water until neutrality, dried over $\mathrm{Na}_{2} \mathrm{SO}_{4}$, filtered and evaporated to give 11 (317.8 mg, 95\%). ${ }^{1} \mathrm{H}-\mathrm{NMR} \delta: 4.60$ (sex, $1 \mathrm{H}$, $\mathrm{J}=6.1, \mathrm{H}-13$ ), 3.53 and 3.51 (two s, 2 x 3H, 2 x COOMe), 3.10 (d, 1H, J=6.2, $\mathrm{H}-7 \beta$-epoxide), 3.00 (sb, 1H, H-7 $\alpha$-epoxide), $2.00-1.78(\mathrm{~m}, 1 \mathrm{H}), 1.81$ and 1.79 (each s, $2 \times \mathrm{H}, 2 \times$ OOCMe), $1.60-1.05$ (m, 13H), 0.97 (d, 3H, J=6.1, Me-16), 0.66 and 0.62 (each s, 3 x 3H, Me-18, Me-19 and Me-20); ${ }^{13} \mathrm{C}-\mathrm{NMR}$

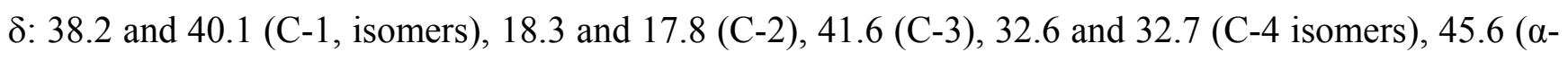
isomer) and 47.1 ( $\beta$-isomer) (C-5), $20.7(\mathrm{C}-6), 57.5$ ( $\alpha$-isomer) and 59.4 ( $\beta$-isomer) $(\mathrm{C}-7), 58.8(\alpha$ isomer) and 60.2 ( $\beta$-isomer) (C-8), 49.1 ( $\beta$-isomer) and 53.5 ( $\alpha$-isomer) $(\mathrm{C}-9), 34.7$ and $35.6(\mathrm{C}-10$ isomers), 22.6 (C-11), 34.9 (C-12), 70.4 (C-13), 21.1 (C-16), 170.2 and 170.7 (C-17 isomers), 32.4 and 33.0 (C-18 isomers), 21.7 (C-19), 14.2 and 14.9 (C-20 isomers), 51.9 and 52.1 (2XCOOMe), 170.2 and 170.7 (2XMeCOO), 19.8 (MeCOO); IR cm ${ }^{-1}$ : 2930, 1731, 1715, 1461, 1251, 1142. 


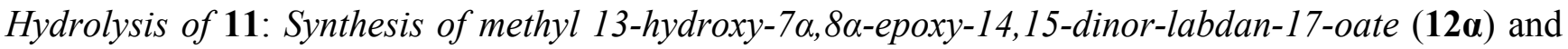

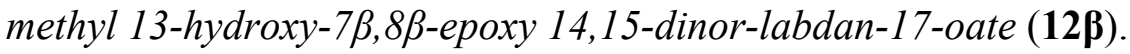

To a solution of $11(250 \mathrm{mg}, 0.68 \mathrm{mmol})$ in methanol $(12 \mathrm{~mL})$ was added $\mathrm{K}_{2} \mathrm{CO}_{3}(150 \mathrm{mg})$. The reaction mixture was stirred at room temperature for $1 \mathrm{~h}$, water was added and the mixture extracted with ether, washed with $2 \mathrm{~N} \mathrm{HCl}$ and $\mathrm{H}_{2} \mathrm{O}$, dried, filtered and evaporated yielding $212 \mathrm{mg}(85 \%)$ of 12 .

Compound 12a: ${ }^{1} \mathrm{H}-\mathrm{NMR} \delta$ : 3.72 (s, 3H, COOMe), 3.71 (m, 1H, H-13), 3.22 (sb, 1H, H-7), 2.14 (dd, $1 \mathrm{H}, \mathrm{J}=4.3,14.4, \mathrm{H}-6), 1.80-1.00$ (m, 13H), 1.14 (d, 3H, J=6.1, Me-16), 0.87 (s, 3H,Me-19), 0.86 (s, $3 \mathrm{H}, 18$ ) and 0.83 (s, 3H, Me-20); ${ }^{13} \mathrm{C}-\mathrm{NMR} \delta: 38.5$ (C-1), 18.6 (C-2), 41.8 (C-3), 33.0 (C-4), 45.9 (C5), 20.1 (C-6), 57.8 (C-7), 59.1 (C-8), 53.8 (C-9), 35.0 (C-10), 21.9 (C-11), 38.3 (C-12), 67.7 (C-13), $23.6(\mathrm{C}-16), 171.1(\mathrm{C}-17), 32.6(\mathrm{C}-18), 21.8(\mathrm{C}-19) 14.6(\mathrm{C}-20), 52.2(\mathrm{COOMe}) ;[\alpha]_{\mathrm{D}}{ }^{20}+6.9(c 0.4 \mathrm{in}$ $\left.\mathrm{CHCl}_{3}\right) ; \mathrm{IR} \mathrm{cm}{ }^{-1}: 3491,2981,1732,1450,1375,1167,1026$.

Compound 12ק: ${ }^{1} \mathrm{H}-\mathrm{NMR} \delta: 3.72$ (m, 1H, H-13), 3.71 (s, 3H, COOMe), 3.30 (d, 1H, J=6.3, H-7), 2.10 -0.90 (m, 14H), 1.16 (d, 3H, J=6.2, Me-16); 0.87 (s, 3H, Me-19); 0.84 (s, 3H, Me-18) and 0.82 (s, 3H, Me-20); ${ }^{13} \mathrm{C}-\mathrm{NMR} \delta: 38.5$ (C-1), 18.0 (C-2), 41.9 (C-3), 32.9 (C-4), 47.7 (C-5), 21.0 (C-6), 59.7 (C-7), 60.5 (C-8), 49.4 (C-9), 35.9 (C-10), 23.2 (C-11), 40.6 (C-12), 68.1 (C-13), 23.4 (C-16), 172.7 (C-17), 33.2 (C-18), 21.9 (C-19), 15.2 (C-20), 52.3 (COOMe); $\mathrm{IR} \mathrm{cm}^{-1}$ : 3486, 2985, 1736, 1455, 1371, 1164, 1026.

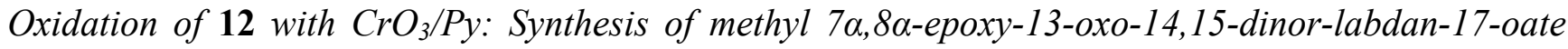

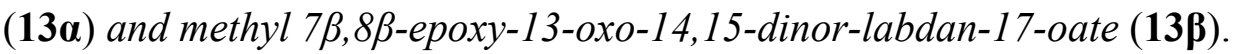

Pyridine $(1 \mathrm{~mL})$ and dry $\mathrm{CH}_{2} \mathrm{Cl}_{2}(3 \mathrm{~mL})$ were placed in a $50 \mathrm{~mL}$ Erlenmeyer flask externally cooled with ice. $\mathrm{CrO}_{3}(660 \mathrm{mg}, 0.65 \mathrm{mmol})$ was added in small portions with stirring until a think yellow paste was obtained. This was allowed to reach room temperature and was stirred for $15 \mathrm{~min}$ under $\mathrm{N}_{2}$. Following this 12 (324.0 mg, $\left.1.0 \mathrm{mmol}\right)$ dissolved in dry $\mathrm{CH}_{2} \mathrm{Cl}_{2}(3 \mathrm{~mL})$ was added and the mixture was stirred vigorously for 1.5 hours. The mixture was filtered and chromatographed on silicagel, yielding $265.7 \mathrm{mg}$ (82\%) of 13. In some fractions the $\alpha$ isomer is the major compound and in other fractions, it is the $\beta$ isomer.

Compound 13a: ${ }^{1} \mathrm{H}-\mathrm{NMR} \delta$ : 3.62 (s, 3H, COOMe), 3.14 (sb, 1H, H-7), 2.35 - 2.20 (m, 1H, H-6), 2.01 (s, 3H, Me-16), $1.90-0.85$ (m, 13H), 0.78 (s, 3H, Me-19), 0.77 (s, 3H, Me-18) and 0.73 (s, 3H, Me20); ${ }^{13} \mathrm{C}-\mathrm{NMR} \delta: 38.1$ (C-1), 17.9 (C-2), 41.6 (C-3), 32.7 (C-4), 45.7 (C-5), 18.3 (C-6), 57.5 (C-7), 58.7 (C-8), 53.1 (C-9), 34.8 (C-10), 21.6 (C-11), 42.2 (C-12), 207.6 (C-13), 29.8 (C-16), 170.5 (C-17), 32.4 (C-18), 21.6 (C-19), 14.2 (C-20), 52.2 (COOMe); IR cm${ }^{-1}:$ 2926, 1732, 1712, 1461, 1282, 1158 , 1062, 897. 
Compound 13ß: ${ }^{1} \mathrm{H}-\mathrm{NMR} \delta: 3.73$ (s, 3H, COOMe), 3.31 (d, 1H, J=6.4, H-7), $2.55-2.40$ (m, 1H, H6), $2.11(\mathrm{~s}, 3 \mathrm{H}, \mathrm{Me}-16), 2.10-0.90(\mathrm{~m}, 13 \mathrm{H}), 0.88$ (s, 3H, Me-19), 0.85 (s, 3H, Me-18) and 0.82 (s, 3H, Me-20); ${ }^{13} \mathrm{C}-\mathrm{NMR}$ 8: 40.5 (C-1), 18.0 (C-2), 41.9 (C-3), 32.9 (C-4), 46.9 (C-5), 20.9 (C-6), 59.9 (C-7), 60.1 (C-8), 49.4 (C-9), 36.0 (C-10), 20.9 (C-11), 42.6 (C-12), 208.2 (C-13), 29.7 (C-16), 172.7 (C-17), 33.3 (C-18), 21.9 (C-19), 15.2 (C-20), 53.5 (COOMe); IR cm ${ }^{-1}$ : 2950, 1733, 1714, 1275, 1163 , 1053.

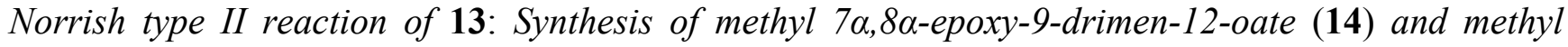
7 $\beta, 8 \beta$-epoxy-9-drimen-12-oate (15).

A solution of $13(250.0 \mathrm{mg}, 0.77 \mathrm{mmol})$ in dry hexane $(250 \mathrm{~mL})$ was placed in a quartz flask and a stream of dry $\mathrm{N}_{2}$ was bubbled through. The solution was irradiated with UV light (Hanau TQ-150, high pressure) for $90 \mathrm{~min}$. Removal of solvent afforded a yellow oil which was purified by chromatography on silica-gel eluting with $98: 2$ hexane-EtOAc to yield $23.3 \mathrm{mg}(9.3 \%)$ of $\mathbf{1 4}, 56.3 \mathrm{mg}$ (22.5\%) of $\mathbf{1 5}$ and $97.5 \mathrm{mg}$ (39\%) of the starting material $\mathbf{1 3 .}$

Compound 14: ${ }^{1} \mathrm{H}-\mathrm{NMR}$ 8: 5.25 (s, 1H, H-11a), 5.13 (s, 1H, H-11b), 3.76 (s, 3H, COOMe), 3.50 (t, $1 \mathrm{H}, \mathrm{J}=1.9, \mathrm{H}-7), 2.20$ (ddd, 1H, J=1.9, 4.3, $15.1 \mathrm{~Hz}, \mathrm{H}-6 \mathrm{a}), 1.80$ (m, 2H, H-6b and H-1), 1.53 (m, 1H), $1.44-1.40$ (m, 3H), 1.20 (dd, 1H, J=4.3, $13.1 \mathrm{~Hz}, \mathrm{H}-5), 1.12$ (m, 1H, H-3), 1.05 (s, 3H, Me-15), 0.90 (s, 3H, Me-14), and 0.85 (s, 3H, Me-13); ${ }^{13} \mathrm{C}-\mathrm{NMR}$ 8: 36.4 (C-1), 18.4 (C-2), 41.8 (C-3), 33.0 (C-4), 41.4 (C-5), 22.2 (C-6), 58.5 (C-7), 57.7 (C-8), 150.8 (C-9), 37.0 (C-10), 114.9 (C-11), 170.0 (C-12), 32.8 (C-13), 22.4 (C-14), 20.2 (C-15), 52.6 (COOMe); $[\alpha]_{\mathrm{D}}^{20}+97.8$ (c 0.5 in $\left.\mathrm{CHCl}_{3}\right) ; \mathrm{IR} \mathrm{cm}^{-1}: 3098$, 1743, 1633, 1373, 1242, 1159, 1047, 902; MS m/z (EI $\left.{ }^{+}\right) 264$ (M+ , 8\%), 263 (3), 249 (49), 232 (26), 217 (35), 205 (22), 189 (46), 175 (29), 161 (74), 154 (30), 147 (59), 135 (88), 121 (69), 107 (87), 105 (92), 91 (100), 79 (80); HRMS (CI) for $\mathrm{C}_{16} \mathrm{H}_{24} \mathrm{O}_{3}\left(\mathrm{MH}^{+}\right)$: Calcd 264.1725; Found 264.1736.

Compound 15: ${ }^{1} \mathrm{H}-\mathrm{NMR} \delta: 5.33$ (s, 1H, H-11a), 5.19 (s, 1H, H-11b), 3.79 (s, 3H, COOMe), 3.57 (d, 1H, J=6.2, H-7), 2.13 (ddd, 1H, J=5.0, 6.2, $15.1 \mathrm{~Hz}, \mathrm{H}-6 \mathrm{a}), 1.87$ (m, 2H, H-6b and H-1), $1.52-1.38$ (m, 4H), $1.22(\mathrm{dd}, 1 \mathrm{H}, \mathrm{J}=5.0,13.2, \mathrm{H}-5), 1.13(\mathrm{~m}, 1 \mathrm{H}), 1.09$ (s, 3H, Me-15), 0.89 (s, 3H, Me-13) and 0.86 (s, 3H, Me-14); ${ }^{13} \mathrm{C}-N M R$ 8: 38.5 (C-1), 18.5 (C-2), 41.8 (C-3), 33.4 (C-4), 48.2 (C-5), 21.3 (C6), 60.6 (C-7), 60.2 (C-8), 151.1 (C-9), 37.6 (C-10), 115.0 (C-11), 170.0 (C-12), 32.8 (C-13), 21.7 (C14), 22.7 (C-15), 52.4 (COOMe); $[\alpha]_{\mathrm{D}}{ }^{20}+73.5$ (c 0.5 in $\left.\mathrm{CHCl}_{3}\right)$; IR cm ${ }^{-1}: 3096,2923,1744,1635$, 1275, 1244, 1151, 1043, 902; MS m/z (EI $\left.{ }^{+}\right) 264$ ( $\left.\mathrm{M}^{+}, 3 \%\right), 263$ (3), 249 (49), 232 (26), 217 (35), 205 (22), 189 (46), 175 (29), 161 (74), 154 (30), 147 (59), 135 (88), 121 (69), 107 (87), 105 (92), 91 (100), 79 (80); HRMS (CI) for $\mathrm{C}_{16} \mathrm{H}_{24} \mathrm{O}_{3}\left(\mathrm{MH}^{+}\right)$: Calcd 264.1725; Found 264.1737. 
Treatment of 1a with m-CPBA: Synthesis of methyl 7,8( $\alpha+\beta)$ epoxy-15-hydroxylabdan-17-oate (16).

Compound 1a (490 mg, $1.5 \mathrm{mmol})$ was dissolved in $\mathrm{CH}_{2} \mathrm{Cl}_{2}(10 \mathrm{~mL})$ and $m$-CPBA (380 mg, 2.2 mmol) was added. The mixture was stirred at $40^{\circ} \mathrm{C}$ and monitored by TLC. After $4 \mathrm{~h}$ the reaction was complete and the solvent evaporated. Work-up afforded $480 \mathrm{mg}$ of crude product that after chromatography on silica-gel gave $323 \mathrm{mg}(67.3 \%)$ of 16 in the 7:3 hexane/EtOAc fractions. ${ }^{1} \mathrm{H}-\mathrm{NMR}$ $\delta$ : 3.68 (s, 3H, COOMe), 3.64 (m, 2H, H-15), 3.26 (d, 1H, J=6.3, H-7 $\beta$-epoxide), 3.18 (sb, 1H, H-7 $\alpha$ epoxide), $2.09-1.88(\mathrm{~m}, 3 \mathrm{H}), 1.82-0.90$ (m, 14H), 0.89 (d, 3H, J=6.7, Me-16), 0.84 (s each, 2 x 3H, Me-18 and Me-19) and 0.80 (s, 3H, Me-20); ${ }^{13} \mathrm{C}-\mathrm{NMR} \delta$ ( $\alpha$-epoxide): $38.4(\mathrm{C}-1), 18.0(\mathrm{C}-2), 41.8$ (C3), 32.8 (C-4), 45.7 (C-5), 20.9 (C-6), 57.7 (C-7), 59.0 (C-8), 54.2 (C-9), 35.0 (C-10), 24.6 (C-11), 36.2 (C-12), 30.1 (C-13), 40.5 (C-14), 60.9 (C-15), 19.6 (C-16), 171.4 (C-17), 32.6 (C-18), 21.9 (C19), 15.2 (C-20), 52.1 (COOMe); ${ }^{13} \mathrm{C}-\mathrm{NMR} \delta$ ( $\beta$-epoxide): 39.2 (C-1), 18.6 (C-2), 41.9 (C-3), 33.2 (C4), 48.4 (C-5), 21.5 (C-6), 59.6 (C-7), 60.7 (C-8), 49.4 (C-9), 35.9 (C-10), 24.6 (C-11), 36.6 (C-12), 29.9 (C-13), 40.5 (C-14), 60.9 (C-15), 19.7 (C-16), 172.7 (C-17), 32.8 (C-18), 21.8 (C-19), 14.6 (C20), 52.1 (COOMe); IR cm ${ }^{-1}: 3445,1732,1457,1283,1063,758$; HRMS (CI) for $\mathrm{C}_{21} \mathrm{H}_{36} \mathrm{O}_{4}\left(\mathrm{MH}^{+}\right)$: Calcd 352.2614; Found 352.2625.

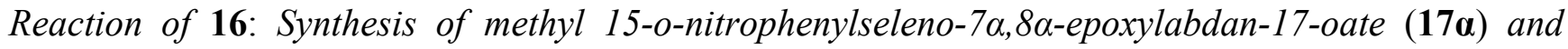
methyl 15-o-nitrophenylseleno-7 $\beta, 8 \beta$-epoxylabdan-17-oate (17及)

To a solution of $16(450 \mathrm{mg}, 1.3 \mathrm{mmol})$ in dry THF $(3.0 \mathrm{~mL})$ was added $o$ nitrophenylselenocyanate $(363.3 \mathrm{mg}, 1.6 \mathrm{mmol})$ and stirred under inert atmosphere for $20 \mathrm{~min}$ at $48^{\circ}$ C. $\mathrm{n}-\mathrm{Bu}_{3} \mathrm{P}(322.9 \mathrm{mg}, 1.6 \mathrm{mmol})$ was added to reactional mixture, stirred for $48 \mathrm{~min}$, the solvent was removed at reduced pressure and the residue chromatographed yielding with hexane/EtOAc 95:5, $338.0 \mathrm{mg}(75 \%)$ of $\mathbf{1 7 .}$

Compound 17a: ${ }^{1} \mathrm{H}-\mathrm{NMR} \delta$ : 8.27 (d, 1H, J=8.1, H-3'), 7.60 - 7.18 (m, 3H, H-4', H-5' and H-6'), 3.67 (s, 3H, COOMe), 3.21 (sb, 1H, H-7), 2.89 (m, 2H, H-15), $2.24-1.90$ (m, 3H), $1.85-0.90$ (m, 14H), 0.94 (d, 3H, J=6.1, Me-16), 0.86 (s each, 2 x 3H, Me-19 and Me-18) and 0.83 (s, 3H, Me-20); ${ }^{13} \mathrm{C}-$ NMR 8: 38.7 (C-1), 18.7 (C-2), 42.0 (C-3), 33.1 (C-4), 46.2 (C-5), 21.7 (C-6), 57.9 (C-7), 59.2 (C-8), 54.7 (C-9), 35.2 (C-10), 22.1 (C-11), 35.0 (C-12), 34.1 (C-13), 35.9 (C-14), 23.9 (C-15), 19.6 (C-16), 171.0 (C-17), 32.7 (C-18), 21.9 (C-19), 14.6 (C-20), 52.2 (COOMe), 134.8 (C-1'), 146.9 (C-2'), 126.5 (C-3'), 129.1 (C-4'), 133.6 (C-5'), 125.3 (C-6'); IR cm ${ }^{-1}$ : 2930, 1740, 1596, 1514, 1331, 715, 711.

Compound 17ß: ${ }^{1} \mathrm{H}-\mathrm{NMR} \delta$ : 8.29 (d, 1H, J=8.2, H-3'), 7.40 - 7.30 (m, 3H,H-4', H-5' and H-6'), 3.71 (s, 3H, COOMe), 3.32 (d, 1H, J=6.4, H-7), 2.90 (m, 2H, H-15), $2.10-1.90$ (m, 2H), $1.85-0.90$ (m, 15H), 1.06 (d, 3H, J=6.1, Me-16), 0.90 (s, 3H, Me-19), 0.85 (s, 3H, Me-18) and 0.85 (s, 3H, Me-20); ${ }^{13} \mathrm{C}-\mathrm{NMR}\left(\mathrm{CDCl}_{3}\right)$ 8: 40.7 (C-1), 18.1 (C-2), 42.0 (C-3), 33.3 (C-4), 48.4 (C-5), 21.1 (C-6), 59.8 (C-7), 60.7 (C-8), 49.5 (C-9), 35.9 (C-10), 24.8 (C-11), 34.9 (C-12), 34.1 (C-13), 36.1 (C-14), 23.9 (C-15), 
19.4 (C-16), 172.7 (C-17), 32.9 (C-18), 22.0 (C-19), 15.4 (C-20), 52.3 (COOMe), 134.4 (C-1'), 146.5 (C-2'), 126.5 (C-3'), 129.0 (C-4'), 133.6 (C-5'), 125.3 (C-6'); IR cm' : 3062, 2929, 1733, 1590, 1513, $1332,1037,754$.

Oxidation of $\mathbf{1 7}$ with $\mathrm{H}_{2} \mathrm{O}_{2}$ : Synthesis of methyl 7,8( $\left.\alpha+\beta\right)$-epoxy-14-labden-17-oate (18).

To a solution of $17(330.0 \mathrm{mg}, 0.61 \mathrm{mmol})$ in THF $(5 \mathrm{~mL})$ was added $30 \% \mathrm{H}_{2} \mathrm{O}_{2}(0.07 \mathrm{~mL}, 42 \mathrm{mg}$, $1.2 \mathrm{mmol}$ ) and the mixture was stirred for $12 \mathrm{~h}$. The solvent was evaporated at reduced pressure and the crude product chromatographed yielding with 98:2 hexane/EtOAc, $284 \mathrm{mg}(86 \%)$ of 18 as a mixture of epoxides. Data is given for a fraction in which the $\beta$-isomer predominates.

Compound 18ß: ${ }^{1} \mathrm{H}-\mathrm{NMR} \delta: 5.63$ (ddd, $\left.1 \mathrm{H}, \mathrm{J}=7.7,10.2,17.5, \mathrm{H}-14\right), 4.93$ (dd, 1H, J=3.1, 17.5, H-15a), 4.91 (dd, 1H, J=3.1, 10.2, H-15b), 3.72 (s, 3H, COOMe), 3.29 (d, 1H, J=6.3, H-7), 2.15 - 1.90 (m, 2H, H-6), $1.85-0.98$ (m, 13H), 0.96 (d, 3H, J=6.7, Me-16), 0.88 (s, 3H, Me-19) and 0.83 (s each, 2X3H, Me-18 and Me-20); ${ }^{13} \mathrm{C}-\mathrm{NMR} \delta: 40.7$ (C-1), 18.1 (C-2), 42.0 (C-3), 32.9 (C-4), 48.0 (C-5), 21.0 (C-6), 59.7 (C-7), 60.6 (C-8), 49.5 (C-9), 35.9 (C-10), 24.9 (C-11), 36.0 (C-12), 38.2 (C-13), 144.3 (C-14), 112.9 (C-15), 20.4 (C-16), 172.7 (C-17), 33.3 (C-18), 22.0 (C-19), 15.3 (C-20), 52.3 (COOMe); IR $\mathrm{cm}^{-1}: 3074,2926,1733,1649,1460,1241$.

Wacker oxidation of 18: Synthesis of methyl 7,8( $\alpha+\beta)$-epoxy-14-oxolabdan-17-oate (19).

To a solution of $\mathrm{PdCl}_{2}(64 \mathrm{mg}, 0.36 \mathrm{mmol})$ and $\mathrm{CuCl}(1.7 \mathrm{~g}, 1.8 \mathrm{mmol})$ in DMF $(8 \mathrm{ml})$ and $\mathrm{H}_{2} \mathrm{O}(1$ $\mathrm{mL})$ was activated for $30 \mathrm{~min}$ with $\mathrm{O}_{2}$. A solution of $18(550 \mathrm{mg}, 1.8 \mathrm{mmol})$ in DMF $(7 \mathrm{~mL})$ was added and stirred with $\mathrm{O}_{2}$ atmosphere at room temperature for $36 \mathrm{~h}$. Then the reaction mixture was added a cooled solution of $3 \mathrm{~N} \mathrm{HCl}$, extracted with $\mathrm{CH}_{2} \mathrm{Cl}_{2}(3 \times 30 \mathrm{~mL})$ and washed successively with $10 \% \mathrm{NaHCO}_{3}$ and water, dried, filtered, evaporated and chromatographed (eluting with 9:1 hexane/EtOAc) to give $440 \mathrm{mg}(80 \%)$ of 19. A fraction collected during this $\mathrm{CC}$ was purified to give $4 \%$ of the $\alpha$-isomer of 19 , which was used for characterization purposes.

Compound 19a: ${ }^{1} \mathrm{H}-\mathrm{NMR} \delta$ : 3.76 (s, 3H,COOMe), 3.23 (sb, 1H, H-7), 2.47 (sex, 1H, J=6.6, H-13), 2.11 (s, 3H, Me-15), $1.84-0.90$ (m, 14H), 1.06 (d, 3H, J=6.6, Me-16), 0.86 (s each, 2X3H, Me-19 and Me-18) and 0.84 (s, 3H, Me-20); ${ }^{13} \mathrm{C}-\mathrm{NMR}$ 8: 38.5 (C-1), 18.6 (C-2), 41.9 (C-3), 33.0 (C-4), 46.0 (C5), 21.9 (C-6), 57.8 (C-7), 59.0 (C-8), 54.4 (C-9), 35.1 (C-10), 21.9 (C-11), 32.3 (C-12), 47.4 (C-13), 212.1 (C-14), 28.0 (C-15), 16.4 (C-16), 170.8 (C-17), 32.6 (C-18), 21.9 (C-19), 14.5 (C-20), 52.3 (COOMe); $m / z\left(\mathrm{EI}^{+}\right) 350\left(\mathrm{M}^{+}, 10 \%\right), 318$ (24), 300 (55), 285 (55), 261 (73), 257 (100), 233 (59), 229 (27), 219 (23), 201 (67), 177 (15), 163 (27), 151 (15), 141 (16), 123 (34), 109 (43), 95 (19), 79 (22), 69 (23), 55 (36); $[\alpha]_{\mathrm{D}}{ }^{20}+11.7\left(c 0.5\right.$ in $\left.\mathrm{CHCl}_{3}\right)$; IR cm ${ }^{-1}: 2926,1731,1718,1461,1282,1158,769$; HRMS (CI) for $\mathrm{C}_{21} \mathrm{H}_{34} \mathrm{O}_{4}\left(\mathrm{MH}^{+}\right)$: Calcd 350.2457; Found 350.2468. 
Reaction of $\mathbf{1 9}$ with m-CPBA: Synthesis of $\mathbf{1 1 .}$

Compound 19 (300 mg, $0.86 \mathrm{mmol}$ ) was dissolved in anhydrous $\mathrm{CH}_{2} \mathrm{Cl}_{2}(10 \mathrm{~mL})$ and $m$-CPBA $\left(276 \mathrm{mg}, 1.6 \mathrm{mmol}\right.$ ) was added. The mixture was stirred at $30^{\circ} \mathrm{C}$ and monitored by TLC. After $5 \mathrm{~h}$ the reaction was complete and the solvent evaporated. Work-up afforded $290 \mathrm{mg}$ of crude product that after chromatography on silica-gel gave in the 9:1 hexane/EtOAc fractions $264 \mathrm{mg}(88 \%)$ of $\mathbf{1 1}$ as an epoxide mixture.

Reaction of 20: Synthesis of 17-acetoxy-15-o-nitrophenylseleno-7-labdene (21).

To a solution of $20(450 \mathrm{mg}, 1.3 \mathrm{mmol})$ in dry THF $(3.0 \mathrm{~mL})$ was added $o$ nitrophenylselenocyanate $(363.3 \mathrm{mg}, 1.6 \mathrm{mmol})$ and the mixture was stirred under an inert atmosphere for $20 \mathrm{~min}$ at $48^{\circ} \mathrm{C}$. Then $\mathrm{n}-\mathrm{Bu}_{3} \mathrm{P}(322.9 \mathrm{mg}, 1.6 \mathrm{mmol})$ was added to the mixture, which ws stirred for $48 \mathrm{~min}$, then the solvent was removed under reduced pressure and the residue chromatographed (95:5 hexane/EtOAc) yielding $382.5 \mathrm{mg}(85 \%)$ of $21 .{ }^{1} \mathrm{H}-\mathrm{NMR} \delta: 8.29$ (d, 1H, J=8.2, H-3'), $7.55-7.26$ (m, 3H, H-4', H-5' and H-6'), 4.57 (d, 1H, J=12.1, H-17a), 4.38 (d, 1H, J=12.1, H-17b), 2.85 (m, 2H, H15), 2.03 (s, 3H, OOCMe), $2.00-0.95(\mathrm{~m}, 17 \mathrm{H}), 0.97$ (d, 3H, J=5.7, Me-16), 0.88 (s, 3H, Me-19), 0.85 (s, 3H, Me-18) and 0.75 (s, 3H, Me-20); ${ }^{13} \mathrm{C}-\mathrm{NMR}$ 8: 39.0 (C-1), 18.7 (C-2), 42.1 (C-3), 32.9 (C4), 49.6 (C-5), 23.8 (C-6), 129.1 (C-7), 134.0 (C-8), 52.5 (C-9), 36.7 (C-10), 23.9 (C-11), 38.3 (C-12), 34.4 (C-13), 34.8 (C-14), 23.9 (C-15), 19.4 (C-16), 67.8 (C-17), 33.0 (C-18), 21.8 (C-19), 13.6 (C-20), 170.6 (MeCOO), 21.2 (Me-COO), 134.7 (C-1'), 146.7 (C-2'), 126.4 (C-3'), 129.1 (C-4'), 133.6 (C-5'), 125.2 (C-6'); IR $\mathrm{cm}^{-1}:$ 2930, 1733, 1591, 1515, 1332, 1247, 737.

Oxidation of $\mathbf{2 1}$ with $\mathrm{H}_{2} \mathrm{O}_{2}$ : Synthesis of 17-acetoxy-7,14-labdadiene (22).

To a solution of $21(330 \mathrm{mg}, 0.61 \mathrm{mmol})$ in THF $(5 \mathrm{~mL})$ was added $30 \% \mathrm{H}_{2} \mathrm{O}_{2}(0.07 \mathrm{~mL}, 42 \mathrm{mg}$, $1.2 \mathrm{mmol}$ ) and this mixure was stirred for $12 \mathrm{~h}$. The solvent was evaporated under reduced pressure and the crude product chromatographed (95:5 hexane/EtOAc) yielding $297 \mathrm{mg}(90 \%)$ of $22 .{ }^{1} \mathrm{H}-\mathrm{NMR}$ 8: 5.71 (m, 1H, H-7), 5.58 (ddd, 1H, J=7.7, 10.3, 17.1, H-14), 4.89 (dd, 1H, J=2.1, 17.1, H-15a), 4.86 (dd, 1H, J=2.1, 17.1, H-15b), 4.50 (d, 1H, J=12.1, H-17a), 4.30 (d, 1H, J=12.1, H-17b), 1.99 (s, 3H, OOCMe), $1.95-0.80$ (m, 15H), 0.92 (d, 3H, J=6.7, Me-16), 0.83 (s, 3H, Me-19), 0.80 (s, 3H, Me-18) and 0.69 (s, 3H, Me-20); ${ }^{13} \mathrm{C}-\mathrm{NMR}$ 8: 38.9 (C-1), 18.6 (C-2), 42.0 (C-3), 32.8 (C-4), 49.5 (C-5), 23.6 (C-6), 128.5 (C-7), 134.1 (C-8), 52.3 (C-9), 36.6 (C-10), 24.0 (C-11), 38.3 (C-12), 38.5 (C-13), 144.1 (C-14), 112.8 (C-15), 20.4 (C-16), 67.7 (C-17), 32.9 (C-18), 21.7 (C-19), 13.4 (C-20), 170.5 (MeCOO), 21.0 (Me-COO); $[\alpha]_{\mathrm{D}}^{20}-3.9$ (c 0.5 in $\mathrm{CHCl}_{3}$ ); IR cm${ }^{-1}: 3076,1742,1640,1240,911$. 
Wacker oxidation of 22: Synthesis of $\mathbf{2 3}$.

A solution of $\mathrm{PdCl}_{2}(64 \mathrm{mg}, 0.34 \mathrm{mmol})$ and $\mathrm{CuCl}(1.6 \mathrm{~g}, 1.6 \mathrm{mmol})$ in $\mathrm{DMF}(8 \mathrm{~mL})$ and $\mathrm{H}_{2} \mathrm{O}(1$ $\mathrm{mL}$ ) was activated for $30 \mathrm{~min}$ with $\mathrm{O}_{2}$. A solution of $22(520 \mathrm{mg}, 1.6 \mathrm{mmol})$ in DMF $(7 \mathrm{~mL})$ was then added and the mixture stirred with an $\mathrm{O}_{2}$ atmosphere at room temperature for $36 \mathrm{~h}$, then it was added to a cooled solution of $3 \mathrm{~N} \mathrm{HCl}$, extracted with $\mathrm{CH}_{2} \mathrm{Cl}_{2}(3 \times 30 \mathrm{~mL})$ and washed successively with $10 \%$ $\mathrm{NaHCO}_{3}$ and water, dried, filtered, evaporated and chromatographed (9:1 hexane/EtOAc) to give 416 $\mathrm{mg}(80 \%)$ of $\mathbf{2 3}$.

Treatment of $\mathbf{2 3}$ with m-CPBA: Synthesis of 13,17-diacetoxy-7,8-epoxy-14,15-dinor-labdane (24).

Compound 23 (300 mg, $0.86 \mathrm{mmol})$ was dissolved in anhydrous $\mathrm{CH}_{2} \mathrm{Cl}_{2}(10 \mathrm{~mL})$ and $m$-CPBA (276 mg, $1.6 \mathrm{mmol}$ ) was added. The mixture was stirred at $40^{\circ} \mathrm{C}$ and monitored by TLC. After $4 \mathrm{~h}$ the reaction was complete and the solvent evaporated. Work-up afforded $290 \mathrm{mg}$ of crude product that after chromatography on silica-gel giving $264 \mathrm{mg}$ (88\%) of 24 (as a mixture of epoxides) in the 9:1 hexane/EtOAc fractions. ${ }^{1} \mathrm{H}-\mathrm{NMR} \delta: 4.95-4.71$ (m, 1H, H-13), 4.20 (d, 1H, J=12.1, H-17a), 4.03 (d, 1H, J=12.1, H-17b), 3.22 (d, 1H, J=6.1, H-7 $\beta$-isomer), 3.14 (sb, 1H, H-7 $\alpha$-isomer), 2.04 and 1.99 (s each, 2X3H, 2X-OOCMe), $1.85-0.75(\mathrm{~m}, 14 \mathrm{H}), 1.19$ (d, 3H, J=6.2, Me-16), 0.83 (s, 3H, Me-19), 0.82 (s, 3H, Me-18) and 0.72 (s, 3H, Me-20); ${ }^{13} \mathrm{C}-\mathrm{NMR} \delta: 38.0$ and 38.4 (C-1, isomers), 18.5 (C-2), 41.9 (C-3), 32.9 (C-4), 45.6 (C-5), 20.3 (C-6), 57.7 (C-7), 58.6 (C-8), 54.1 (C-9), 35.6 (C-10), 22.1 (C11), 38.0 and 38.4 (C-12, isomers), 70.8 (C-13), 20.8 (C-16), 67.1 (C-17), 32.5 (C-18), 21.8 (C-19), 14.0 (C-20), 170.3 and 170.9 (2 x MeCOO), 21.2 (2 x Me-COO); IR cm ${ }^{-1}: 2925,1724,1246,1024$.

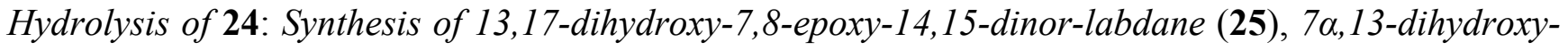

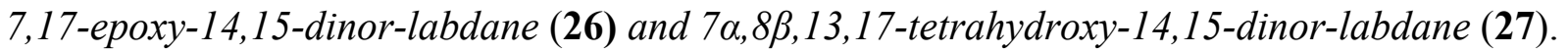

Method A: To a solution of $24(98.5 \mathrm{mg}, 0.26 \mathrm{mmol})$ in methanol $(4 \mathrm{~mL})$ was added $\mathrm{K}_{2} \mathrm{CO}_{3}(120 \mathrm{mg})$. The reaction mixture was stirred at room temperature for $10 \mathrm{~h}$, water was added and the mixture extracted with ether washed with $2 \mathrm{~N} \mathrm{HCl}$ and $\mathrm{H}_{2} \mathrm{O}$, dried, filtered, evaporated and chromatographed yielding $59.1 \mathrm{mg}$ of $\mathbf{2 5}+\mathbf{2 6}(60 \%)$, and $29.6 \mathrm{mg}$ of $\mathbf{2 7}(30 \%)$.

Method B: To a solution of $24(110 \mathrm{mg}, 0.29 \mathrm{mmol})$ was added a solution of $4 \% \mathrm{NaOH}$ in methanol (6 $\mathrm{mL}$ ). The reaction mixture was stirred at room temperature for $5 \mathrm{~h}$, water was added and the mixture extracted with ether, washed with $2 \mathrm{~N} \mathrm{HCl}$ and $\mathrm{H}_{2} \mathrm{O}$, dried, filtered, evaporated and chromatographed yielding $33 \mathrm{mg}$ (30\%) of 25, $16.5 \mathrm{mg}$ (15\%) of $\mathbf{2 6}$ and $36.3 \mathrm{mg}(33 \%)$ of 27.

Compound 25: ${ }^{1} \mathrm{H}-\mathrm{NMR} \delta: 3.83(\mathrm{~d}, 1 \mathrm{H}, \mathrm{J}=12.2, \mathrm{H}-17 \mathrm{a}), 3.70$ (m, 1H, H-13), 3.65 (d, 1H, J=12.2 Hz, $\mathrm{H}-17 \mathrm{~b}), 3.30$ (m, 1H, H-7, $\alpha$ and $\beta$ isomer), $1.90-0.90$ (m, 14H), 1.42 (d, 3H, J=6.8, Me-16), 0.87 (s, $3 \mathrm{H}, \mathrm{Me}-19$ ) and 0.85 (s each, $2 \times 3 \mathrm{H}, \mathrm{Me}-18$ and Me-20); ${ }^{13} \mathrm{C}-\mathrm{NMR} \delta: 38.5$ and 40.9 (C-1, isomers), 
18.0 and 18.4 (C-2, isomers), 41.6 and 41.9 (C-3, isomers), 32.9 (C-4), 45.6 (C-5), 20.4 (C-6), 57.0 (C7), 58.6 (C-8), 54.3 (C-9), 35.8 (C-10), 22.8 (C-11), 38.5 and 40.9 (C-12 isomer), 67.8 and 67.9 (C-13 isomers), 23.5 and 23.9 (C-16 isomers), 65.4 (C-17), 32.6 and 33.1 (C-18 isomers), 21.6 and 21.9 (C19 isomers), 13.9 and 14.2 (C-20 isomers); IR cm ${ }^{-1}$ : 3420, 2924, 1474, 1253, 1090, 1070.

Compound 26: ${ }^{1} \mathrm{H}-\mathrm{NMR} \delta: 3.70$ (m, 1H, H-13), 3.34 (t, 1H, J=2.9, H-7), 2.68 (d, 1H, J=4.3, H-17a), 2.44 (d, 1H, J=4.3, H-17b), $1.89-0.95$ (m, 14H), 1.16 (d, 3H, J=6.7, Me-16), 0.87 (s, 3H, Me-19) and 0.85 (s each, $2 \mathrm{X} 3 \mathrm{H}, \mathrm{Me}-18$ and Me-20); ${ }^{13} \mathrm{C}-\mathrm{NMR} \delta$ : 38.5 and 40.9 (C-1 isomers), 18.0 and 18.4 (C-2 isomers), 41.6 and 41.9 (C-3 isomers), 32.9 (C-4), 46.8 (C-5), 27.7 (C-6), 73.5 (C-7), 61.2 (C-8), 46.9 (C-9), 35.8 (C-10), 22.8 (C-11), 38.5 and 40.9 (C-12 isomers), 67.8 and 67.9 (C-13 isomers), 23.5 and 23.9 (C-16 isomers), 48.6 (C-17), 32.6 and 33.1 (C-18 isomers), 21.6 and 21.9 (C-19 isomers), 13.9 and 14.2 (C-20 isomers); IR cm ${ }^{-1}: 3420,2924,1472,1252,1090,1070$.

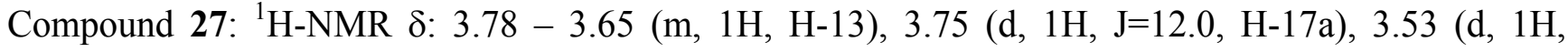
$\mathrm{J}=12.0, \mathrm{H}-17 \mathrm{~b}$ ), 3.17 (sb, 1H, H-7), $1.90-1.00$ (m, 14H), 1.15 (d, 3H, J=6.4, Me-16), 0.85 (s, 3H, Me19), 0.83 (s, 3H, Me-19) and 0.80 (s, 3H, Me-20); ${ }^{13} \mathrm{C}-\mathrm{NMR} \delta: 38.7$ (C-1), 18.2 (C-2), 42.0 (C-3), 33.1 (C-4), 46.6 (C-5), 25.6 (C-6), 70.7 (C-7), 76.5 (C-8), 50.2 (C-9), 35.8 (C-10), 20.9 (C-11), 42.0 (C-12), 68.6 (C-13), 23.7 (C-16), 66.3 (C-17), 33.1 (C-18), 21.7 (C-19), 15.1 (C-20); IR cm ${ }^{-1}: 3420,1080$.

Oxidation of 1a: Synthesis of 7-labden-17-methoxycarbonyl-15-oic acid (28).

$\mathrm{CrO}_{3}(620 \mathrm{mg}, 6.2 \mathrm{mmol})$ was added to $90 \%$ acetic acid $(10.0 \mathrm{~mL})$. After stirring for $15 \mathrm{~min}, \mathbf{1 a}$ $(1.4 \mathrm{~g}, 4.17 \mathrm{mmol})$ in $\mathrm{CH}_{2} \mathrm{Cl}_{2}(10 \mathrm{~mL})$ were added and stirring was continued at $40^{\circ} \mathrm{C}$ for $40 \mathrm{~h} . \mathrm{MeOH}$ was added and stirred for $30 \mathrm{~min}$, the solvent removed in vacuo. The reaction product was extracted with ether, the ether solution was washed with a solution of $4 \% \mathrm{NaOH}$ and the $\mathrm{pH}$ of the aqueous solution was adjusted to a value of 2 with $\mathrm{HCl}$ and the acidic products were recovered by extraction with ether. The solution of the acidic products was dried with anhydrous $\mathrm{Na}_{2} \mathrm{SO}_{4}$. Solvent removal and chromatography on silica-gel gave $860 \mathrm{mg}(60 \%)$ of $\mathbf{2 8}$ in the $6: 4$ hexane/EtOAc fractions. ${ }^{1} \mathrm{H}-\mathrm{NMR} \delta$ : $9.70(\mathrm{sb}, 1 \mathrm{H}, \mathrm{COOH}), 6.62(\mathrm{~m}, 1 \mathrm{H}, \mathrm{H}-7), 3.62(\mathrm{~s}, 3 \mathrm{H}, \mathrm{COOMe}), 2.42-1.70(\mathrm{~m}, 4 \mathrm{H}), 1.68-0.90(\mathrm{~m}$, $13 \mathrm{H}$ ), 0.94 (d, 3H, J=6.9, Me-16), 0.87 (s, 3H, Me-19), 0.84 (s, 3H, Me-18) and 0.79 (s, 3H, Me-20); ${ }^{13} \mathrm{C}-\mathrm{NMR}$ 8: 39.5 (C-1), 18.5 (C-2), 42.0 (C-3), 32.7 (C-4), 49.4 (C-5), 23.9 (C-6), 137.2 (C-7), 135.3 (C-8), 50.9 (C-9), 36.9 (C-10), 25.6 (C-11), 38.1 (C-12), 31.0 (C-13), 41.3 (C-14), 179.7 (C-15), 19.7 (C-16), 170.7 (C-17), 33.1 (C-18), 21.9 (C-19), 14.3 (C-20), 51.3 (COOMe); IR cm ${ }^{-1}: 3500$ - 2500, $2929,1710,1645,1254$. 
Epoxidation of 28: Synthesis of 29.

To a solution of $28(846 \mathrm{mg}, 2.41 \mathrm{mmol})$ in dry $\mathrm{CH}_{2} \mathrm{Cl}_{2}(5 \mathrm{~mL}), m$-CPBA (800 mg, $\left.4.6 \mathrm{mmol}\right)$ was added and the mixture stirred at $40^{\circ} \mathrm{C}$. After $30 \mathrm{~h}$, the solvent was removed and ether was added, the organic phase was washed with $40 \% \mathrm{Na}_{2} \mathrm{~S}_{2} \mathrm{O}_{3}, 10 \% \mathrm{Na}_{2} \mathrm{CO}_{3}$ and water to neutrality, dried over $\mathrm{Na}_{2} \mathrm{SO}_{4}$, filtered and evaporated to give 29 (508 $\mathrm{mg}, 60 \%$ ).

Oxidation of 16: Synthesis of 7,8( $\alpha+\beta)$ epoxylabdan-17-methoxycarbonyl-15-oic acid (29).

Method $\mathrm{A}: \mathrm{CrO}_{3}(235 \mathrm{mg}, 2.3 \mathrm{mmol})$ was added to $90 \%$ acetic acid $(5.0 \mathrm{~mL})$. After stirring for $15 \mathrm{~min}$, 16 (330 mg, $0.9 \mathrm{mmol})$ in $\mathrm{CH}_{2} \mathrm{Cl}_{2}(5 \mathrm{~mL})$ and glacial acetic acid $(2 \mathrm{~mL})$ were added and stirring was continued at room temperature for $8 \mathrm{~h}$. $\mathrm{MeOH}$ was added and the solvent removed in vacuo. Work-up afforded $320 \mathrm{mg}$ of crude product that after chromatography on silica-gel gave $181.5 \mathrm{mg}(55 \%)$ of 29 in the EtOAc fractions.

Method B: To a stirred solution of PDC (1.2 mg, $3.3 \mathrm{mmol})$ in DMF (12.0 mL) was added 16 (340 mg, $1.0 \mathrm{mmol}$ ). After $40 \mathrm{~h}$ at room temperature the solvent is evaporated, extracted with ether, washed with 7-10 vol of water and dried over $\mathrm{Na}_{2} \mathrm{SO}_{4}$. The residue was chromatographed on silica-gel affording $889 \mathrm{mg}$ (74\%) of 29 in the EtOAc fractions.

Compound 29: ${ }^{1} \mathrm{H}-\mathrm{NMR} \delta: 3.70$ (s, 3H, COOMe), 3.28 (d, 1H, J=6.3, H-7 $\beta$ ), 3.26 (sb, 1H, H-7 2 ), 2.40 $-2.00(\mathrm{~m}, 3 \mathrm{H}), 1.95-1.00(\mathrm{~m}, 14 \mathrm{H}), 0.96$ (d, 3H, J=6.7, Me-16), 0.88 (s each, 2X3H, Me-18 and Me19) and 0.85 (s, 3H, Me-20); ${ }^{13} \mathrm{C}-\mathrm{NMR} \delta: 38.6$ (C-1), 18.6 (C-2), 42.0 (C-3), 33.0 (C-4), 46.0 (C-5), 21.6 (C-6), 57.8 (C-7), 59.0 (C-8), 54.5 (C-9), 35.1 (C-10), 21.9 (C-11), 36.0 (C-12), 30.6 (C-13), 41.2 (C-14), 178.4 (C-15), 19.8 (C-16), 170.8 (C-17), 32.6 (C-18), 21.8 (C-19), 14.5 (C-20), 52.1 (COOMe); IR cm ${ }^{-1}: 3550$ - 2500, 1730, 1700, 1089, 758; HRMS (CI) for $\mathrm{C}_{21} \mathrm{H}_{34} \mathrm{O}_{5}\left(\mathrm{MH}^{+}\right)$: Calcd 366.4917; Found 366.4928.

Reaction of 28: Synthesis of methyl 15-nor-7 $(\alpha+\beta)$-acetoxy-8-labden-17-oate (30) and methyl 15-nor7,13-labdadien-17-oate (31).

To a solution of $28(343 \mathrm{mg}, 0.98 \mathrm{mmol})$ in dry $\mathrm{C}_{6} \mathrm{H}_{6}(10 \mathrm{~mL})$ was added dry pyridine $(0.1 \mathrm{~mL})$ under a $\mathrm{N}_{2}$ atmosphere and the mixture was stirred at room temperature. After 15 min, dry $(\mathrm{AcO})_{2} \mathrm{Cu}$ $(60 \mathrm{mg}, 0.33 \mathrm{mmol})$ was added and the reaction mixture was heated at $80^{\circ} \mathrm{C}$. $(\mathrm{AcO})_{4} \mathrm{~Pb}(1.280 \mathrm{~g}, 2.9$ mmol) was added in six portions over the next $6 \mathrm{~h}$. The solvent was removed and extracted with ether. The ethereal solution of neutral products was washed with water and dried over $\mathrm{Na}_{2} \mathrm{SO}_{4}$, filtered and evaporated to give $273 \mathrm{mg}$ of crude mixture that by CC afforded $103.2 \mathrm{mg}$ of $\mathbf{3 0}$ and $21.8 \mathrm{mg}$ of $\mathbf{3 1}$. 
Compound 30: ${ }^{1} \mathrm{H}-\mathrm{NMR} \delta: 5.69(\mathrm{t}, 1 \mathrm{H}, \mathrm{J}=8.7, \mathrm{H}-7 \beta), 5.68(\mathrm{~m}, 1 \mathrm{H}, \mathrm{H}-7 \alpha), 3.66(\mathrm{~s}, 2$ x $3 \mathrm{H}, 2 \mathrm{x}$ COOMe), $2.60-2.00(\mathrm{~m}, 4 \mathrm{H}), 1.98$ (s, 2 x 3H, 2 x OOCMe), $1.97-1.10(\mathrm{~m}, 10 \mathrm{H}), 1.09$ (s, 3H, Me20, $\beta$ ), 0.97 (s, 3H, Me-20, $\alpha$ ), 0.87 (d, 2 x 3H, J=6.9, Me-16), 0.84 (d, 2 x 3H, J=6.9, Me-14) and 0.83 (s, 4 x 3H, Me-18 and Me-19); IR cm ${ }^{-1}: 2956,1733,1651,1238$.

Compound 31: ${ }^{1} \mathrm{H}-\mathrm{NMR} \delta$ : 6.61 (m, 1H, H-7), 4.63 (s, 2H, H-14), 3.69 (s, 3H, COOMe), $2.40-1.71$ (m, 6H), $1.68(\mathrm{~s}, 3 \mathrm{H}, \mathrm{Me}-16), 1.62-0.98(\mathrm{~m}, 8 \mathrm{H}), 0.88$ (s, 3H, Me-19), 0.85 (s, 3H, Me-18) and 0.81 (s, 3H, Me-20); ${ }^{13} \mathrm{C}-\mathrm{NMR}\left(\mathrm{CDCl}_{3}\right)$ $\delta: 39.3$ (C-1), 18.5 (C-2), 42.0 (C-3), 32.8 (C-4), 49.4 (C-5), 23.8 (C-6), 136.9 (C-7), 135.4 (C-8), 50.7 (C-9), 36.9 (C-10), 26.8 (C-11), 39.4 (C-12), 146.8 (C-13), 109.3 (C-14), 22.5 (C-16), 169.8 (C-17), 33.1 (C-18), 21.9 (C-19), 14.3 (C-20), 51.3 (COOMe); $[\alpha]_{\mathrm{D}}{ }^{20}-19.0$ (c 0.2 in $\left.\mathrm{CHCl}_{3}\right)$; IR cm ${ }^{-1}: 3079,2960,1720,1652,798$.

Reaction of 31 with $\mathrm{O}_{3}$ and Norrish type II reaction: Synthesis of $\mathbf{5}$.

A solution of $31(100 \mathrm{mg}, 0.3 \mathrm{mmol})$, in $\mathrm{CH}_{2} \mathrm{Cl}_{2}(4 \mathrm{~mL})$ was cooled to $-78^{\circ} \mathrm{C}$ with acetone/Dry Ice. Ozone (about $5.2 \mathrm{~g}$ of $\mathrm{O}_{3} / \mathrm{h}$ ) was bubbled through this solution for $1.5 \mathrm{~min}$. To the cooled reaction mixture $\mathrm{Ph}_{3} \mathrm{P}(164.5 \mathrm{mg}, 0.6 \mathrm{mmol})$ in $\mathrm{CH}_{2} \mathrm{Cl}_{2}(3 \mathrm{~mL})$ was added and then it was gradually allowed to reach room temperature. The solvent was removed under reduced pressure and the residue chromatographed on silica-gel to give $75 \mathrm{mg}$ (75\%) of product. A solution of the reaction product (75 $\mathrm{mg}, 0.24 \mathrm{mmol})$ in dry hexane $(250 \mathrm{~mL})$ was placed in a quartz flask and a stream of dry $\mathrm{N}_{2}$ was bubbled through it. The solution was irradiated with UV light (Hanau TQ-150, high pressure) for $2 \mathrm{~h}$. Removal of solvent afforded a yellow oil which was purified by chromatography on silica-gel eluting with 95:5 hexane/EtOAc to yield $37.5 \mathrm{mg}(50 \%)$ of 5 and $34.2 \mathrm{mg}(45 \%)$ of the starting material.

Hydrolysis of 30: Synthesis of methyl 15-nor-7 $\beta$-hydroxy-8-labden-17-oate (32) and methyl 15-nor-7 $\alpha$ hydroxy-8-labden-17-oate (33).

To a solution of $\mathbf{3 0}(41.4 \mathrm{mg}, 0.12 \mathrm{mmol})$ in methanol $(4 \mathrm{~mL})$ was added $\mathrm{K}_{2} \mathrm{CO}_{3}(60 \mathrm{mg})$. The reaction mixture was stirred at room temperature for $10 \mathrm{~h}$, water was added and the mixture extracted with ether, washed with $2 \mathrm{~N} \mathrm{HCl}$ and $\mathrm{H}_{2} \mathrm{O}$, dried, filtered, evaporated and chromatographed yielding $15.5 \mathrm{mg}$ of $\mathbf{3 2}$ and $10.4 \mathrm{mg}$ of $\mathbf{3 3}$.

Compound 32: ${ }^{1} \mathrm{H}-\mathrm{NMR} \delta: 4.68(\mathrm{t}, 1 \mathrm{H}, \mathrm{J}=8.7, \mathrm{H}-7), 3.76(\mathrm{~s}, 3 \mathrm{H}, \mathrm{COOMe}), 2.62(\mathrm{~m}, 1 \mathrm{H}), 2.20-1.10$ (m, 14H), 1.10 (s, 3H, Me-20), 0.90 (s, 3H, Me-19), 0.89 (d, 3H, J=6.8, Me-16), 0.88 (d, 3H, J=6.8, Me-14) and 0.86 (s, 3H, Me-18); ${ }^{13} \mathrm{C}-\mathrm{NMR} \delta: 40.1$ (C-1), 18.7 (C-2), 41.4 (C-3), 33.0 (C-4), 49.5 (C5), 28.4 (C-6), 69.7 (C-7), 128.6 (C-8), 158.5 (C-9), 40.6 (C-10), 26.6 (C-11), 35.9 (C-12), 29.1 (C13), 22.3 (C-14), 22.3 (C-16), 170.4 (C-17), 33.0 (C-18), 21.7 (C-19), 20.1 (C-20), 51.3 (COOMe); $[\alpha]_{\mathrm{D}}^{20}+38.4\left(c 0.2\right.$ in $\left.\mathrm{CHCl}_{3}\right) ; \mathrm{IR} \mathrm{cm}^{-1}: 3400,2940,1720,1630,1460,1250,1060 ; \mathrm{MS} \mathrm{m} / z\left(\mathrm{EI}^{+}\right) 322$ (M+3\%), 304 (17), 290 (4), 273 (4), 263 (17), 248 (19), 230 (7), 219 (20), 201 (19), 191 (10), 177 
(14), 173 (13), 166 (30), 163 (63), 159 (12), 151 (19), 149 (13), 147 (11), 145 (16), 142 (32), 139 (15), 133 (19), 131 (17), 129 (11), 123 (26), 119 (37), 117 (17), 109 (60), 105 (40), 91 (40), 83 (21), 81 (27), 79 (27), 77 (22), 59 (20), 55 (70), 43 (79), 41 (100); HRMS (CI) for $\mathrm{C}_{20} \mathrm{H}_{34} \mathrm{O}_{3}\left(\mathrm{MH}^{+}\right)$: Calcd 322.2508; Found 322.2520 .

Compound 33: ${ }^{1} \mathrm{H}-\mathrm{NMR}$ 8: 4.44 (m, 1H, H-7), 3.76 (s, 3H, COOMe), $2.61-2.41$ (m, 2H), $1.99-1.10$ (m, 11H), 0.98 (s, 3H, Me-20), 0.96 (d, 3H, J=6.8, Me-16), 0.88 (d, 3H, J=6.8, Me-14) and 0.86 (s, 2X3H, Me-18 and Me-19); ${ }^{13} \mathrm{C}-\mathrm{NMR} \delta: 40.1$ (C-1), 18.9 (C-2), 41.4 (C-3), 33.0 (C-4), 44.9 (C-5), 27.1 (C-6), 65.8 (C-7), 126.3 (C-8), 161.7 (C-9), 41.3 (C-10), 27.2 (C-11), 35.6 (C-12), 29.2 (C-13), 22.3 (C-14), 22.4 (C-16), 170.6 (C-17), 33.1 (C-18), 21.8 (C-19), 18.5 (C-20), $51.4(\mathrm{COOMe}) ;[\alpha]_{\mathrm{D}}{ }^{20}$ + 41.4 (c 0.3 in $\left.\mathrm{CHCl}_{3}\right) ; \mathrm{IR} \mathrm{cm}{ }^{-1}: 3450,2950,1715,1620,1460,1230,1100 ; \mathrm{MS} m / z\left(\mathrm{EI}^{+}\right) 322\left(\mathrm{M}^{+}\right.$, 1\%), 304 (17), 263 (17), 248 (16), 234 (17), 233 (100), 219 (25), 201 (28), 191 (15), 177 (16), 167 (10), 164 (18), 163 (98), 159 (15), 149 (11), 147 (11), 145 (18), 133 (20), 121 (19), 119 (48), 117 (17), 105 (40), 95 (23), 91 (46), 83 (24), 81 (22), 79 (25), 77 (23), 69 (50), 67 (22), 55 (55), 43 (73), 41 (92).

Reaction of 29: Synthesis of methyl 7-oxo-15-nor-8-labden-17-oate (34) and methyl 7 $\beta, 8 \beta$-epoxy-15nor-13-labden-17-oate (35ß).

To a solution of $29(250 \mathrm{mg}, 0.68 \mathrm{mmol})$ in dry $\mathrm{C}_{6} \mathrm{H}_{6}(10 \mathrm{~mL})$ was added dry pyridine $(0.1 \mathrm{~mL})$ under a $\mathrm{N}_{2}$ atmosphere and the mixture was stirred at room temperature. After $15 \mathrm{~min}$, dry $(\mathrm{AcO})_{2} \mathrm{Cu}$ (50 mg, $0.27 \mathrm{mmol}$ ) was added and the reaction mixture is heated to $80^{\circ} \mathrm{C}$. Over the next $6 \mathrm{~h}$ $(\mathrm{AcO})_{4} \mathrm{~Pb}(774 \mathrm{mg}, 1.74 \mathrm{mmol})$ was added in six portions. The solvent was removed and the residue extracted with ether. The ethereal solution was washed with a solution of $4 \% \mathrm{NaOH}$ for extraction of the unreacted acid $29(44.2 \mathrm{mg})$. The solution of neutral products was washed with water and dried over $\mathrm{Na}_{2} \mathrm{SO}_{4}$, filtered and evaporated to give $195 \mathrm{mg}$ of a mixture that by CC afforded $155 \mathrm{mg}(62 \%)$ of $\mathbf{3 4}$ and $30 \mathrm{mg}(12 \%)$ of $\mathbf{3 5}$.

Compound 34: ${ }^{1} \mathrm{H}-\mathrm{NMR} \delta: 3.76$ (s, 3H, COOMe), $2.60-2.00(\mathrm{~m}, 4 \mathrm{H}), 1.90-1.12(\mathrm{~m}, 13 \mathrm{H}), 1.12(\mathrm{~s}$, $3 \mathrm{H}, \mathrm{Me}-20$ ), 0.87 (d, 3H, J=7.2, Me-16), 0.86 (s each, 2X3H, Me-18 and Me-19) and 0.85 (d, 3H, $\mathrm{J}=7.2$, Me-14); ${ }^{13} \mathrm{C}-\mathrm{NMR}$ 8: 38.9 (C-1), 18.3 (C-2), 41.0 (C-3), 33.2 (C-4), 49.8 (C-5), 34.9 (C-6), 196.4 (C-7), 132.1 (C-8), 167.9 (C-9), 40.5 (C-10), 28.1 (C-11), 34.9 (C-12), 29.0 (C-13), 22.0 (C-14), 22.1 (C-16), 172.7 (C-17), 32.4 (C-18), 21.3 (C-19), 18.3 (C-20), 52.1 (COOMe); [ $\alpha]_{\mathrm{D}}{ }^{20}+22.3$ (c 0.4 in $\left.\mathrm{CHCl}_{3}\right) ; \mathrm{IR} \mathrm{cm}^{-1}: 2956,1750,1667,1583,1462,1348,1242,1144,1095,1022,801 ; \mathrm{MS} m / z\left(\mathrm{EI}^{+}\right)$ $320\left(\mathrm{M}^{+}, 0.8 \%\right), 288$ (25), 273 (14), 245 (100), 217 (41), 189 (27), 175 (29), 161 (25), 149 (32), 135 (23), 121 (22), 109 (39), 91 (31), 79 (22), 77 (17); HRMS (CI) for $\mathrm{C}_{20} \mathrm{H}_{32} \mathrm{O}_{3}\left(\mathrm{MH}^{+}\right.$): Calcd 320.2351; Found 320.2363 . 
Compound 35ß: ${ }^{1} \mathrm{H}-\mathrm{NMR} \delta$ : 4.65 (s, 1H, H-14a), 4.63 (s, 1H, H-14b), 3.70 (s, 3H, COOMe), 3.28 (d, 1H, J=6.4, H-7), $2.22-0.99$ (m, 14H), 1.67 (s, 3H, Me-16), 0.86 (s, 3H, Me-19), 0.83 (s, 3H, Me-19) and 0.81 (s, 3H, Me-20); ${ }^{13} \mathrm{C}-\mathrm{NMR}$ 8: 40.5 (C-1), 18.1 (C-2), 42.0 (C-3), 32.9 (C-4), 47.9 (C-5), 25.8 (C-6), 59.7 (C-7), 60.6 (C-8), 49.4 (C-9), 35.9 (C-10), 21.0 (C-11), 37.3 (C-12), 146.0 (C-13), 109.8 (C-14), 22.5 (C-16), 172.5 (C-17), 33.2 (C-18), 22.0 (C-19), 15.3 (C-20), 52.3 (COOMe); IR cm ${ }^{-1}$ : 3070, 2931, 1740, 1650, 1454, 1381, 1283, 1161, 1054, 891, 769; MS m/z (EI $\left.{ }^{+}\right) 320\left(\mathrm{M}^{+}, 0.8 \%\right), 281$ (8), 243 (7), 234 (9), 219 (49), 207 (23), 193 (20), 135 (26), 123 (44), 109 (100), 95 (65), 93 (43), 81 (83), 79 (45); HRMS (CI) for $\mathrm{C}_{20} \mathrm{H}_{32} \mathrm{O}_{3}\left(\mathrm{MH}^{+}\right)$: Calcd 320.2351; Found 320.2362.

Reaction of $\mathbf{3 5}$ with $\mathrm{O}_{3}$ : Synthesis of $\mathbf{1 3 .}$

A solution of $35(220 \mathrm{mg}, 0.69 \mathrm{mmol})$ in $\mathrm{CH}_{2} \mathrm{Cl}_{2}(6 \mathrm{~mL})$, was cooled to $-78^{\circ} \mathrm{C}$ with acetone/dry ice. Ozone (about $5.2 \mathrm{~g}$ of $\mathrm{O}_{3} / \mathrm{h}$ ) was bubbled through this solution for $8 \mathrm{~min}$. To the cooled reaction mixture $\mathrm{Ph}_{3} \mathrm{P}(230 \mathrm{mg}, 0.90 \mathrm{mmol})$ in $\mathrm{CH}_{2} \mathrm{Cl}_{2}(6 \mathrm{~mL})$ was added and the mixture was gradually allowed to reach room temperature. The solvent was then removed under reduced pressure and the residue chromatographed on silica-gel affording $16.7 \mathrm{mg}$ (7.6\%) of $\mathbf{3 5}$ and $158.4 \mathrm{mg}$ (72\%) of $\mathbf{1 3}$.

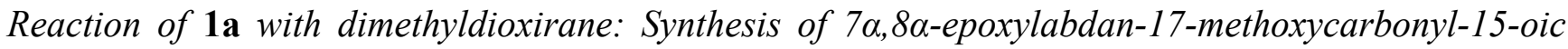
$\operatorname{acid}(\mathbf{2 9 \alpha})$.

To a solution of 1a $(196 \mathrm{mg}, 0.58 \mathrm{mmol})$ in acetone $(5 \mathrm{~mL})$ was added dimethyldioxirane $(8 \mathrm{~mL}$, $0.1 \mathrm{M})$. The reaction is carried out at room temperature. After $20 \mathrm{~min}$, the solvent was removed and the residue chromatographed on silica-gel (elution with 7:3 hexane/EtOAc) affording $88.2 \mathrm{mg} \mathrm{(45 \% )} \mathrm{of}$ 29a. ${ }^{1} \mathrm{H}-\mathrm{NMR}$ 8: 3.72 (s, 3H, COOMe), 3.22 (sb, 1H, H-7), 2.40 - 1.00 (m, 17H), 0.96 (d, 3H, J=6.7, Me-16), 0.89 (s, 3H, Me-19), 0.88 (s, 3H, Me-18) and 0.85 (s, 3H, Me-20); ${ }^{13} \mathrm{C}-\mathrm{NMR} \delta: 38.6$ (C-1), 18.6 (C-2), 42.0 (C-3), 33.0 (C-4), 46.0 (C-5), 21.6 (C-6), 57.8 (C-7), 59.0 (C-8), 54.5 (C-9), 35.1 (C10), 21.9 (C-11), 36.0 (C-12), 30.6 (C-13), 41.2 (C-14), 178.4 (C-15), 19.8 (C-16), 170.8 (C-17), 32.6 (C-18), 21.8 (C-19), 14.5 (C-20), 52.1 (COOMe); IR cm ${ }^{-1}: 3380$ - 2600, 1736, 1712, 1437, 1279, $1161,1054,755$.

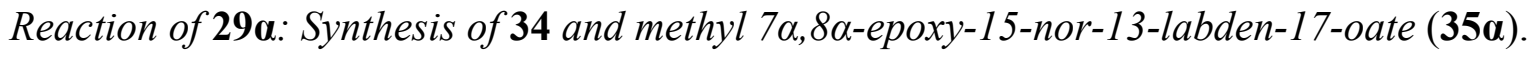

To a solution of $29 \boldsymbol{\alpha}(250 \mathrm{mg}, 0.68 \mathrm{mmol})$ in dry $\mathrm{C}_{6} \mathrm{H}_{6}(10 \mathrm{~mL})$ was added dry pyridine $(0.1 \mathrm{~mL})$ under a $\mathrm{N}_{2}$ atmosphere and the mixture was stirred at room temperature. After $15 \mathrm{~min}$, dry $(\mathrm{AcO})_{2} \mathrm{Cu}$ (42 $\mathrm{mg}, 0.23 \mathrm{mmol}$ ) was added and the reaction mixture was heated to $80^{\circ} \mathrm{C}$. Over $6 \mathrm{~h}$ additional $(\mathrm{AcO})_{4} \mathrm{~Pb}(882.8 \mathrm{mg}, 2.0 \mathrm{mmol})$ was added in six portions. The solvent was removed and the residue extracted with ether. The ethereal solution was washed with a solution of $4 \% \mathrm{NaOH}$ to extract the unreacted acid $29 \alpha(25 \mathrm{mg})$. The solution of neutral products was washed with water and dried over $\mathrm{Na}_{2} \mathrm{SO}_{4}$, filtered and evaporated to give $220 \mathrm{mg}$ of a mixture that after CC afforded $149 \mathrm{mg}(60 \%)$ of 
34 and $30.1 \mathrm{mg}(12 \%)$ of $\mathbf{3 5 \alpha} .{ }^{1} \mathrm{H}-\mathrm{NMR} \delta: 4.71$ (s, 1H, H-14a), 4.68 (s, 1H, H-14b), 3.73 (s, 3H, COOMe), 3.23 (sb, 1H, H-7), 2.20 - 1.00 (m, 14H), 1.63 (s, 3H, Me-16), 0.89 (s, 3H, Me-19), 0.87 (s, 3H, Me-18) and 0.85 (s, 3H, Me-20); ${ }^{13} \mathrm{C}-\mathrm{NMR}$ 8: 38.5 (C-1), 18.6 (C-2), 42.0 (C-3), 33.0 (C-4), 46.0 (C-5), 22.0 (C-6), 57.8 (C-7), 59.0 (C-8), 53.3 (C-9), 35.3 (C-10), 22.3 (C-11), 36.9 (C-12), 145.2 (C13), 110.7 (C-14), 22.2 (C-16), 171.0 (C-17), 32.6 (C-18), 21.9 (C-19), 14.6 (C-20), 52.2 (COOMe); IR $\mathrm{cm}^{-1}: 3073,2927,1733,1649,1437,1277,1053,886,768$.

Reaction of $\mathbf{3 5 \alpha}$ with $\mathrm{O}_{3}$ : Synthesis of $\mathbf{1 3 \alpha}$.

A solution of $35 \boldsymbol{\alpha}(100 \mathrm{mg}, 0.31 \mathrm{mmol})$ in $\mathrm{CH}_{2} \mathrm{Cl}_{2}(5 \mathrm{~mL})$, was cooled to $-78^{\circ} \mathrm{C}$. Ozone (about 5.2 $\mathrm{g}$ of $\left.\mathrm{O}_{3} / \mathrm{h}\right)$ was bubbled through this solution for $8 \mathrm{~min}$. To the cooled reaction mixture $\mathrm{Ph}_{3} \mathrm{P}(158.4$ $\mathrm{mg}, 0.62 \mathrm{mmol})$ in $\mathrm{CH}_{2} \mathrm{Cl}_{2}(6 \mathrm{~mL})$ was added and the mixture was gradually allowed to reach room temperature. The solvent was then removed under reduced pressure and the residue chromatographed on silica-gel affording $6.0 \mathrm{mg}(6 \%)$ of $\mathbf{3 5} \boldsymbol{\alpha}$ and $72.0 \mathrm{mg}(72 \%)$ of $\mathbf{1 3 \alpha}$.

Norrish type II reaction of $\mathbf{1 3 \alpha}$ : Synthesis of $\mathbf{1 4 .}$

A solution of $\mathbf{1 3 \alpha}(100 \mathrm{mg}, 0.31 \mathrm{mmol})$ in dry hexane $(250 \mathrm{~mL})$ was placed in a quartz flask and a stream of dry $\mathrm{N}_{2}$ was bubbled through. The solution was irradiated with UV light (Hanau TQ-150, high pressure) for $90 \mathrm{~min}$. Removal of the solvent afforded a yellow oil which was purified by chromatography on silica-gel eluting with 98:2 hexane/EtOAc, to yield $28.5 \mathrm{mg}(28 \%)$ of $\mathbf{1 4}$ and 40.0 $\mathrm{mg}(40 \%)$ of the starting material $\mathbf{1 3 \alpha}$.

\section{References}

1. Ware, W. G. "Pesticides, Theory and Application", W. H. Freeman and Company: San Francisco, 1983.

2. Van Beek, T. A.; De Groot, A. Recl. Trav. Chim. Pays Bas, 1986, 105, 513; and references cited therein.

3. Knusli, E. "Industrial aspects of the practical use of natural products or derivatives in the protection of crops". In "Natural Products and the Protection of Plants", Marini-Bettolo, G. B. (Ed), Pontificia Accademia Scientiarium Scripta Varia, 1977, 41, 755.

4. Butterworth, J. H.; Morgan, E. D. J. Chem. Soc., Chem. Commum., 1968, 23.

5. Anderson, J. C.; Blaney, W. M.; Cole, M. D.; Fellons, L. L.; Ley, S. V.; Shephard R. N.; Simmonds, M. S. J. Tetrahedron Lett., 1989, 30, 4737.

6. Kubo, I.; Lee, Y. W.; Pettei, M. J.; Pilkiewicz F.; Nakanishi, K. J. J. Chem. Soc., Chem. Coтmum., 1976, 1013.

7. Barnes, C. S.; Loder, J. W. Aust. J. Chem., 1962, 15, 32.

8. Nakanishi, K.; Rube, I. Israel J. Chemistry, 1977, 16, 28. 
9. Singhal, S.; Mathur, S. C. Chem. Ind., 1993, 112.

10. Urones, J. G.; Marcos, I. S.; Pérez, B. G.; Díez, D.; Lithgow, A. M.; Gómez, P. M.; Basabe P.; Garrido, N. M. Tetrahedron, 1994, 50, 10995.

11. Urones, J. G.; Marcos, I. S.; Martín, D. D.; Brito Palma, F. M.; Rodilla, J. M. Phytochemistry, 1987, 26, 3037.

12. Urones, J. G.; Marcos I. S.; Martín, D. D. Tetrahedron, 1988, 44, 112.

13. Grieco P. A.; Jaw, J. Y. J. Org. Chem., 1981, 46, 1215.

14. Mizuno, M.; Cava M. P.; Gabrito, A. F. J. Org. Chem., 1976, 41, 1485.

15. Tsuji, J.; Shimizu I.; Yamamoto, K.Tetrahedron Lett., 1976, 34, 2975.

16. Lamers, Y. M.; Rusu, G.; Wijnberg J. B.; de Groot, A. Tetrahedron, 2003, 59, 9361.

17. Kubo, I.; Mura, I.; Pettei, M. J.; Lee, Y. W.; Pikiewicz, F.; Nakanishi, K. Tetrahedron Lett., 1997, 52, 4553.

18. Nakamo T.; Maillo, M. A. Synth. Commun. 1981, 11, 463.

19. Urones, J. G.; Marcos, I. S.; Gómez Pérez, B.; Lithgow, A. M.; Díez, D.; Gómez, P. M.; Basabe, P.; Garrido, N. M. Tetrahedron, 1995, 51, 1845.

20. Payne, J. J. Org. Chem., 1962, 27, 3819.

21. Corey, E. J.; Schmidt, G.Tetrahedron Lett., 1979, 37, 399.

22. Bacha J. D.; Cochi, J. K. Tetrahedron, 1968, 24, 2215.

23. Adam, W.; Chan, Y. Y.; Cremer, D.; Gauss, J.; Scheutzow D.; Schindler, M. J. Org. Chem., 1987, 52,2800 .

24. Urones, J. G.; Marcos, I. S.; Pérez, B. G.; Lithgow, A. M.; Díez, D.; Basabe P.; Gómez, P. M. Tetrahedron Lett., 1994, 35, 3781.

Sample availability: Small amounts (mgs) of the final compounds are available from the authors.

(C) 2004 by MDPI (http:www.mdpi.org). Reproduction is permitted for noncommercial purposes. 